\title{
Finite Element Methods for Linear Elasticity
}

Richard S. Falk*

Department of Mathematics - Hill Center

Rutgers, The State University of New Jersey

110 Frelinghuysen Rd., Piscataway, NJ 08854-8019

falk@math.rutgers . edu

Key words: mixed method, finite element, elasticity

1 Introduction $\ldots \ldots \ldots \ldots \ldots \ldots \ldots \ldots \ldots \ldots \ldots \ldots \ldots$

$2 \quad$ Finite element methods with strong symmetry .......... 4

$3 \quad$ Exterior calculus on $\mathbb{R}^{n} \ldots \ldots \ldots \ldots \ldots \ldots \ldots \ldots \ldots \ldots \ldots$

4 Basic finite element spaces and their properties .......... 14

5 Mixed formulation of the equations of elasticity with

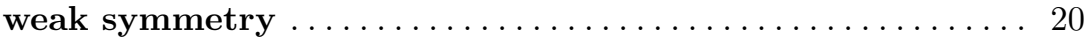

6 From the de Rham complex to an elasticity complex with

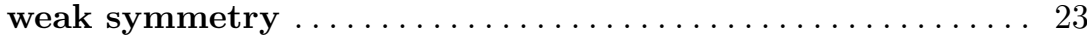

7 Well-posedness of the weak symmetry formulation of elasticity ................................... 24

8 Conditions for stable approximation schemes.......... 25

$9 \quad$ Stability of finite element approximation schemes ........ 28

10 Refined error estimates . . . . . . . . . . . . . . . . . . . . . . 29

11 Examples of stable finite element methods for the weak

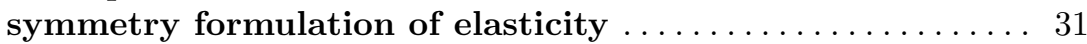

References ................................ 37

\section{Introduction}

The equations of linear elasticity can be written as a system of equations of the form

\footnotetext{
* This work supported by NSF grants DMS03-08347 and DMS06-09755. 9/8/06.
} 


$$
A \sigma=\varepsilon(u), \quad \operatorname{div} \sigma=f \quad \text { in } \Omega .
$$

Here the unknowns $\sigma$ and $u$ denote the stress and displacement fields caused by a body force $f$ acting on a linearly elastic body which occupies a region $\Omega \subset \mathbb{R}^{n}$, with boundary $\partial \Omega$. Then $\sigma$ takes values in the space $\mathbb{S}=\mathbb{R}_{\mathrm{sym}}^{n \times n}$ of symmetric $n \times n$ matrices and $u$ takes values in $\mathbb{V}=\mathbb{R}^{n}$. The differential operator $\varepsilon$ is the symmetric part of the gradient, (i.e., $(\varepsilon(u))_{i j}=\left(\partial u_{i} / \partial x_{j}+\right.$ $\left.\partial u_{j} / \partial x_{i}\right) / 2$ ), div denotes the divergence operator, applied row-wise, and the compliance tensor $A=A(x): \mathbb{S} \rightarrow \mathbb{S}$ is a bounded and symmetric, uniformly positive definite operator reflecting the properties of the material at each point. In the isotropic case, the mapping $\sigma \mapsto A \sigma$ has the form

$$
A \sigma=\frac{1}{2 \mu}\left(\sigma-\frac{\lambda}{2 \mu+n \lambda} \operatorname{tr}(\sigma) I\right)
$$

where $\lambda(x), \mu(x)$ are positive scalar coefficients, the Lamé coefficients, and $\operatorname{tr}$ denotes the trace. If the body is clamped on the boundary $\partial \Omega$, then the proper boundary condition for the system 1 is $u=0$ on $\partial \Omega$. For simplicity, this boundary condition will be assumed throughout the discussion here. However, there are issues that arise when other boundary conditions are assumed (e.g.,, traction boundary conditions $\sigma n=0)$. The modifications needed to deal with such boundary conditions are discussed in detail in [9].

In the case when $A$ is invertible, i.e., $\sigma=A^{-1} \varepsilon(u)=C \varepsilon(u)$, then for isotropic elasticity, $C \tau=2 \mu(\tau+\lambda \operatorname{tr} \tau I)$. We may then formulate the elasticity system weakly in the form: Find $\sigma \in L^{2}(\Omega, \mathbb{S}), u \in \stackrel{\circ}{H}^{1}(\Omega ; \mathbb{V})$ such that

$$
\begin{gathered}
\int_{\Omega} \sigma: \tau \mathrm{d} x-\int_{\Omega} C \varepsilon(u): \tau \mathrm{d} x=0, \tau \in L^{2}(\Omega, \mathbb{S}), \\
\int_{\Omega} \sigma: \varepsilon(v) \mathrm{d} x=\int_{\Omega} f \cdot v \mathrm{~d} x, v \in \stackrel{\circ}{H}^{1}(\Omega ; \mathbb{V}),
\end{gathered}
$$

where $\sigma: \tau=\sum_{i, j=1}^{n} \sigma_{i j} \tau_{i j}$. Note that in this case, we may eliminate $\sigma$ completely to obtain the pure displacement formulation: Find $u \in \stackrel{\circ}{H}^{1}(\Omega ; \mathbb{V})$ such that

$$
\int_{\Omega} C \varepsilon(u): \varepsilon(v) \mathrm{d} x=\int_{\Omega} f \cdot v \mathrm{~d} x, v \in \stackrel{\circ}{H}^{1}(\Omega ; \mathbb{V}) .
$$

As the material becomes incompressible, i.e., $\lambda \rightarrow \infty$, this will not be a good formulation, since the operator norm of $C$ is also approaching infinity. Instead, we can consider a formulation involving $u$ and a new variable $p=(\lambda /[2 \mu+$ $n \lambda]) \operatorname{tr} \sigma$. Taking the trace of the equation $A \sigma=\varepsilon(u)$, we find that $\operatorname{div} u=$ $\lambda^{-1} p$. Then we may write $\sigma=2 \mu \varepsilon(u)+p I$, and thus obtain the variational formulation: Find $u \in \stackrel{\circ}{H}^{1}(\Omega ; \mathbb{V}), p \in L_{0}^{2}(\Omega)=\left\{p \in L^{2}(\Omega): \int_{\Omega} p \mathrm{~d} x=0\right\}$, such that

$$
\begin{gathered}
\int_{\Omega} 2 \mu \varepsilon(u): \varepsilon(v) \mathrm{d} x+\int_{\Omega} p \operatorname{div} v \mathrm{~d} x=\int_{\Omega} f \cdot v \mathrm{~d} x, v \in \stackrel{\circ}{H}^{1}(\Omega ; \mathbb{V}), \\
\int_{\Omega} \operatorname{div} u q \mathrm{~d} x=\int_{\Omega} \lambda^{-1} p q \mathrm{~d} x, q \in L_{0}^{2}(\Omega) .
\end{gathered}
$$


This formulation makes sense even for the limit $\lambda \rightarrow \infty$ and in that case gives the stationary Stokes equations. Even in the case of nearly incompressible elasticity, one should apply methods that are stable for the Stokes equations. Since such methods will be considered in other lectures, we will not consider them here. Instead, we now turn to other types of weak formulations involving both $\sigma$ and $u$. One of these is to seek $\sigma \in H(\operatorname{div}, \Omega ; \mathbb{S})$, the space of squareintegrable symmetric matrix fields with square-integrable divergence, and $u \in$ $L^{2}(\Omega ; \mathbb{V})$, satisfying

$$
\begin{gathered}
\int_{\Omega}(A \sigma: \tau+\operatorname{div} \tau \cdot u) \mathrm{d} x=0, \tau \in H(\operatorname{div}, \Omega ; \mathbb{S}), \\
\int_{\Omega} \operatorname{div} \sigma \cdot v \mathrm{~d} x=\int_{\Omega} f \cdot v \mathrm{~d} x, v \in L^{2}(\Omega ; \mathbb{V}) .
\end{gathered}
$$

A second weak formulation, that enforces the symmetry weakly, seeks $\sigma \in$ $H(\operatorname{div}, \Omega ; \mathbb{M}), u \in L^{2}(\Omega ; \mathbb{V})$, and $p \in L^{2}(\Omega ; \mathbb{K})$ satisfying

$$
\begin{gathered}
\int_{\Omega}(A \sigma: \tau+\operatorname{div} \tau \cdot u+\tau: p) \mathrm{d} x=0, \quad \tau \in H(\operatorname{div}, \Omega ; \mathbb{M}), \\
\int_{\Omega} \operatorname{div} \sigma \cdot v \mathrm{~d} x=\int_{\Omega} f \cdot v \mathrm{~d} x, \quad v \in L^{2}(\Omega ; \mathbb{V}), \\
\int_{\Omega} \sigma: q \mathrm{~d} x=0, \quad q \in L^{2}(\Omega ; \mathbb{K}),
\end{gathered}
$$

where $\mathbb{M}$ is the space of $n \times n$ matrices, $\mathbb{K}$ the subspace of skew-symmetric matrices, and the compliance tensor $A(x)$ is now considered as a symmetric and positive definite operator mapping $\mathbb{M}$ into $\mathbb{M}$.

Stable finite element discretizations with reasonable computational complexity based on the variational formulation 2 have proved very difficult to construct. In particular, it is not possible to simply take multiple copies of standard finite elements for scalar elliptic problems, since the resulting stress matrix will not be symmetric. One successful approach has been to use composite elements, in which the approximate displacement space consists of piecewise polynomials with respect to one triangulation of the domain, while the approximate stress space consists of piecewise polynomials with respect to a different, more refined, triangulation $[22,30,24,4]$. In two space dimensions, the first stable finite elements with polynomial shape functions were presented in [10]. The simplest and lowest order spaces in the family of spaces constructed consist of discontinuous piecewise linear vector fields for displacements and a stress space which is locally the span of piecewise quadratic matrix fields and the cubic matrix fields that are divergence-free. Hence, it takes 24 stress and six displacement degrees of freedom to determine an element on a given triangle. A simpler first-order element pair with 21 stress and three displacement degrees of freedom per triangle is also constructed in [10]. All of these elements require vertex degrees of freedom. To obtain simpler elements, the same authors also considered nonconforming elements in [12]. One 
element constructed there approximates the stress by a nonconforming piecewise quadratic with 15 degrees of freedom and approximates the displacement field by discontinuous linear vectors (6 local degrees of freedom). A second element reduces the number of degrees of freedom to 12 and 3, respectively. See also [11] for an overview. In three dimensions, a piecewise quartic stress space is constructed with 162 degrees of freedom on each tetrahedron in [1].

Because of the lack of suitable mixed elasticity elements that strongly impose the symmetry of the stresses, a number of authors have developed approximation schemes based on the weak symmetry formulation 3: see [22], [2], [3], [27], [28], [29], [5], [25], [26], [21]. Although 2 and 3 are equivalent on the continuous level, an approximation scheme based on 3 may not produce a symmetric approximation to the stress tensor, depending on the choices of finite element spaces.

These notes will mainly concentrate on the development and analysis of finite element approximations of the equations of linear elasticity based on the mixed formulation 3 with weak symmetry. Using a generalization of an approach first developed in [8] in two dimensions and [6] in three dimensions, and then expanded further in [9], we establish a systematic way to obtain stable finite element approximation schemes. The families of methods developed in [8] and [6] are the prototype examples and we show that they satisfy the conditions we develop for stability. However, the somewhat more general approach we present here allows us to analyze some of the previously proposed schemes discussed above in the same systematic manner and also leads to a new scheme. Before considering weakly symmetric schemes, we first discuss some methods based on the strong symmetry formulation 2 .

\section{Finite element methods with strong symmetry}

In this section, we consider finite element methods based on the variational formulation 2. Thus, we let $\boldsymbol{\Sigma}_{h} \subset H(\operatorname{div}, \Omega ; \mathbb{S})$ and $\boldsymbol{V}_{h} \subset L^{2}(\Omega ; \mathbb{V})$ and seek $\sigma_{h} \in \boldsymbol{\Sigma}_{h}$ and $u_{h} \in \boldsymbol{V}_{h}$ satisfying

$\int_{\Omega}\left(A \sigma_{h}: \tau+\operatorname{div} \tau \cdot u_{h}\right) \mathrm{d} x=0, \tau \in \boldsymbol{\Sigma}_{h}, \quad \int_{\Omega} \operatorname{div} \sigma_{h} \cdot v \mathrm{~d} x=\int_{\Omega} f \cdot v \mathrm{~d} x, v \in \boldsymbol{V}_{h}$.

This is in a form to which one may apply the standard analysis of mixed finite element theory (e.g., $[14,15,20,18]$. We note that in the case of isotropic elasticity, if we write $\sigma=\sigma_{D}+(1 / n) \operatorname{tr} \sigma I$, where $\operatorname{tr} \sigma_{D}=0$, then $\|\sigma\|_{0}^{2}=$ $\left\|\sigma_{D}\right\|_{0}^{2}+(1 / n)\|\operatorname{tr} \sigma\|_{0}^{2}$ and so

$$
\int_{\Omega} A \sigma: \sigma \mathrm{d} x=\int_{\Omega}\left[\frac{1}{2 \mu} \sigma_{D}: \sigma_{D}+\frac{1}{2 \mu+n \lambda}(\operatorname{tr} \sigma)^{2}\right] \mathrm{d} x .
$$

Thus, this form is not uniformly coercive as $\lambda \rightarrow \infty$. However, for all $\sigma$ satisfying 


$$
\int_{\Omega} \operatorname{tr} \sigma \mathrm{d} x=0, \quad \operatorname{div} \sigma=0,
$$

one can show (cf. [15]) that $\|\operatorname{tr} \sigma\|_{0} \leq C\left\|\sigma_{D}\right\|_{0}$, and hence $(A \sigma, \sigma) \geq$ $\alpha\|\sigma\|_{H(\text { div })}^{2}$ for all $\sigma$ satisfying 4, with $\alpha$ independent of $\lambda$. This is what is needed to satisfy the first Brezzi condition with a constant independent of $\lambda$. A simple result of mixed finite element theory, giving conditions under which the second Brezzi condition is satisfied, and that fits the methods that we will consider here, is the following.

Theorem 2.1 Suppose that for every $\tau \in \boldsymbol{H}^{1}(\Omega)$, there exists $\Pi_{h} \tau \in \boldsymbol{\Sigma}_{h}$ satisfying

$$
\int_{\Omega} \operatorname{div}\left(\tau-\Pi_{h} \tau\right) \cdot v \mathrm{~d} x=0, \quad v \in \boldsymbol{V}_{h}, \quad\left\|\Pi_{h} \tau\right\|_{H(\operatorname{div})} \leq C\|\tau\|_{H(\operatorname{div})} .
$$

Further suppose that for all $\tau \in \boldsymbol{\Sigma}_{h}$ satisfying $\int_{\Omega} \operatorname{div} \tau \cdot v \mathrm{~d} x=0, v \in \boldsymbol{V}_{h}$, that $\operatorname{div} \tau=0$. Then for all $v_{h} \in \boldsymbol{V}_{h}$,

$$
\left\|\sigma-\sigma_{h}\right\|_{0} \leq C\left\|\sigma-\Pi_{h} \sigma\right\|_{0}, \quad\left\|u-u_{h}\right\|_{0} \leq C\left(\left\|u-v_{h}\right\|_{0}+\left\|\sigma-\sigma_{h}\right\|_{0}\right) .
$$

To describe some finite element methods based on the strong symmetry formulation, we let $\mathcal{P}_{k}(X, Y)$ denote the space of polynomial functions on $X$ of degree at most $k$ and taking values in $Y$.

\subsection{Composite elements}

One of the first methods based on the symmetric formulation was the method of [30] analyzed in [24]. We describe below only the triangular element (there was also a similar quadrilateral element). The basic idea is to approximate the stresses by a composite finite element. Starting from a mesh $\mathcal{T}_{h}$ of triangles, one connects the barycenter of each triangle $K$ to the three vertices to form a composite element made up of three triangles, i.e., $K=T_{1} \cup T_{2} \cup T_{3}$. We then define

$$
\begin{gathered}
\boldsymbol{\Sigma}_{h}=\left\{\tau \in H(\operatorname{div}, \Omega ; \mathbb{S}):\left.\tau\right|_{T_{i}} \in \mathcal{P}_{1}\left(T_{i}, \mathbb{S}\right)\right\}, \\
\boldsymbol{V}_{h}=\left\{v \in \boldsymbol{L}^{2}(\Omega):\left.v\right|_{K} \in \mathcal{P}_{1}\left(K, \mathbb{R}^{2}\right\} .\right. \\
\text { Composite } \\
\text { Element }
\end{gathered}
$$

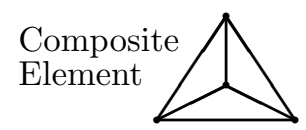

Thus the displacements are defined on the coarse mesh $\mathcal{T}_{h}$. By the definition of $\left.\boldsymbol{\Sigma}_{h}\right|_{K}$, we start from a space of 27 degrees of freedom, on which we impose at most 12 constraints that require that $\tau n$ be continuous across each of the three internal edges of $K$. In fact, these constraints are all independent. Then, a key point is to show that on each $K, \tau$ is uniquely determined by 
the following 15 degrees of freedom (i) the values of $\tau \cdot n$ at two points on each edge of $K$ and (ii) $\int_{K} \tau_{i j} \mathrm{~d} x, i, j=1,2$. It is then easy to check that if $\int_{K} \operatorname{div} \tau \cdot v \mathrm{~d} x=0$ for $v \in \mathcal{P}_{1}\left(K, \mathbb{R}^{2}\right)$, then $\operatorname{div} \tau=0$. If we define $\Pi_{h}$ to correspond to the degrees of freedom, then it is also easy to check that $\int_{K} \operatorname{div}\left(\tau-\Pi_{h} \tau\right) \cdot v \mathrm{~d} x=0$ for $v \in \mathcal{P}_{1}\left(K, \mathbb{R}^{2}\right)$. After establishing the $H(\operatorname{div}, \Omega)$ norm bound on $\Pi_{h} \sigma$, one easily obtains the error estimates:

$$
\left\|\sigma-\sigma_{h}\right\|_{0} \leq C h^{2}\|\sigma\|_{2}, \quad\left\|u-u_{h}\right\|_{0} \leq C h^{2}\left(\|\sigma\|_{2}+\|u\|_{2}\right) .
$$

The use of composite finite elements to approximate the stress tensor was extended to a family of elements in [4]. For $k \geq 2$,

$$
\begin{gathered}
\boldsymbol{\Sigma}_{h}=\left\{\tau \in H(\operatorname{div}, \Omega ; \mathbb{S}):\left.\tau\right|_{T_{i}} \in \mathcal{P}_{k}\left(T_{i}, \mathbb{S}\right)\right\} \\
\boldsymbol{V}_{h}=\left\{v \in \boldsymbol{L}^{2}(\Omega):\left.v\right|_{K} \in \mathcal{P}_{k-1}\left(K, \mathbb{R}^{2}\right)\right\}
\end{gathered}
$$

The space $\boldsymbol{\Sigma}_{h}$ is constructed so that if $\left.\tau \in \boldsymbol{\Sigma}_{h}\right|_{K}$, then $\tau n$ will be continuous across internal edges, and in addition $\operatorname{div} \tau \in \mathcal{P}_{k-1}\left(K, \mathbb{R}^{2}\right)$, i.e., it is a vector polynomial on $K$, not just on each of the $T_{i}$.

The degrees of freedom for an element $\tau \in \boldsymbol{\Sigma}_{h}$ on the triangle $K$ are chosen to be

$$
\begin{gathered}
\int_{e}(\tau n) \cdot p \mathrm{~d} s, \quad p \in \mathcal{P}_{k}\left(e, \mathbb{R}^{2}\right), \quad \text { for each edge } e, \\
\int_{K} \tau: \varrho \mathrm{d} x, \quad \varrho \in \varepsilon\left(\mathcal{P}_{k-1}\left(K, \mathbb{R}^{2}\right)\right)+\operatorname{airy}\left(\lambda_{1}^{2} \lambda_{1}^{2} \lambda_{3}^{2} P_{k-4}(K, \mathbb{R})\right),
\end{gathered}
$$

where the $\lambda_{i}$ are the barycentric coordinates of $K$ and

$$
J \phi \equiv \operatorname{airy} \phi=\left(\begin{array}{cc}
\partial^{2} \phi / \partial y^{2} & -\partial^{2} \phi / \partial x \partial y \\
-\partial^{2} \phi / \partial x \partial y & \partial^{2} \phi / \partial x^{2}
\end{array}\right)
$$

One can show that $\left.\operatorname{dim} \boldsymbol{\Sigma}_{h}\right|_{K}=(3 / 2) k^{2}+(9 / 2) k+6$. In the lowest order case $k=2$, there are 18 edge degrees of freedom and 3 interior degrees of freedom on each macro-triangle $K$. For the general case $k \geq 2$, it is shown that

$$
\begin{gathered}
\left\|u-u_{h}\right\|_{0} \leq C h^{r}\|u\|_{r}, \quad 2 \leq r \leq k, \\
\left\|\sigma-\sigma_{h}\right\|_{0} \leq C h^{r}\|u\|_{r+1}, \quad 1 \leq r \leq k+1, \\
\left\|\operatorname{div}\left(\sigma-\sigma_{h}\right)\right\|_{0} \leq C h^{r}\|\operatorname{div} \sigma\|_{r}, \quad 0 \leq r \leq k .
\end{gathered}
$$

\subsection{Non-composite elements of Arnold and Winther}

We now turn to the more recent methods that produce approximations to both stresses and displacements that are polynomial on each triangle $T \in \mathcal{T}_{h}$ (since there are no macro triangles, we no longer use $K$ to denote a generic triangle). The approach of [10] is based on the use of discrete differential complexes and the close relation between the construction of stable mixed 
finite element methods for the approximation of the Laplacian and discrete versions of the de Rham complex

$$
\mathbb{R} \stackrel{\subset}{\hookrightarrow} C^{\infty}(\Omega) \stackrel{\text { curl }}{\longrightarrow} C^{\infty}\left(\Omega ; \mathbb{R}^{2}\right) \stackrel{\text { div }}{\longrightarrow} C^{\infty}(\Omega) \rightarrow 0 .
$$

If we assume that $\Omega$ is simply-connected, this sequence is exact (i.e., the range of each map is the kernel of the following one). As discussed later in this paper, many of the standard spaces leading to stable mixed finite element methods for Laplace's equation have the property that the following diagram commutes

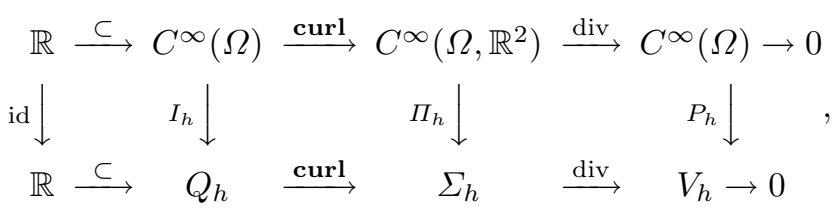

where $I_{h}, \Pi_{h}, P_{h}$ are the natural interpolation operators into the corresponding finite element spaces $Q_{h}, \Sigma_{h}$, and $V_{h}$. For example, the simplest case is when $Q_{h}$ is the space of continuous piecewise linear functions, $\Sigma_{h}$ the space of lowest order Raviart-Thomas elements, and $V_{h}$ the space of piecewise constants. The right half of the commuting diagram, involving $\Pi_{h}$ and $P_{h}$ is a key result in establishing the second Brezzi stability condition. See [7],and [9] for further discussion of this idea.

The starting point of [10] is that there is also an elasticity differential complex, which summarizes important aspects of the structure of the plane elasticity system, i.e.,

$$
P_{1}(\Omega) \stackrel{\subset}{\longrightarrow} C^{\infty}(\Omega) \stackrel{J}{\longrightarrow} C^{\infty}(\Omega, \mathbb{S}) \stackrel{\operatorname{div}}{\longrightarrow} C^{\infty}\left(\Omega, \mathbb{R}^{2}\right) \rightarrow 0 .
$$

Again assuming that $\Omega$ is simply-connected, this sequence is also exact. Thus this sequence encodes the fact that every smooth vector-field is the divergence of a smooth symmetric matrix-field, that the divergence-free symmetric matrix-fields are precisely those that can be written as the Airy stress-field associated to some scalar potential, and that the only potentials for which the corresponding Airy stress vanishes are the linear polynomials. The result stated above is in terms of smooth functions, but analogous results hold with less smoothness. For example, the sequence

$$
P_{1}(\Omega) \stackrel{\subset}{\longrightarrow} H^{2}(\Omega) \stackrel{J}{\rightarrow} H(\operatorname{div}, \Omega ; \mathbb{S}) \stackrel{\operatorname{div}}{\longrightarrow} L^{2}\left(\Omega, \mathbb{R}^{2}\right) \rightarrow 0
$$

is also exact. The well-posedness of the continuous problem, i.e., that for every $f \in L^{2}\left(\Omega, \mathbb{R}^{2}\right)$, there exists a unique $(\sigma, u) \in H(\operatorname{div}, \Omega ; \mathbb{S}) \times L^{2}\left(\Omega, \mathbb{R}^{2}\right)$ which is a critical point of (1.1), follows from this.

Just as there is a close relation between the construction of stable mixed finite element methods for the approximation of the Laplacian and discrete versions of the de Rham complex, there is also a close relation between mixed finite elements for linear elasticity and discretization of the elasticity complex, 
given above. The stable pairs of finite element spaces $\left(\boldsymbol{\Sigma}_{h}, \boldsymbol{V}_{h}\right)$ introduced in [10] have the property that $\operatorname{div} \boldsymbol{\Sigma}_{h}=\boldsymbol{V}_{h}$, i.e., the short sequence

$$
\boldsymbol{\Sigma}_{h} \stackrel{\operatorname{div}}{\longrightarrow} \boldsymbol{V}_{h} \rightarrow 0
$$

is exact. Moreover, if there are projections $P_{h}: C^{\infty}\left(\Omega, \mathbb{R}^{2}\right) \mapsto \boldsymbol{V}_{h}$ and $\Pi_{h}$ : $C^{\infty}(\Omega, \mathbb{S}) \mapsto \boldsymbol{\Sigma}_{h}$ defined by the degrees of freedom that determine the finite element spaces, it can be shown that the following diagram commutes:

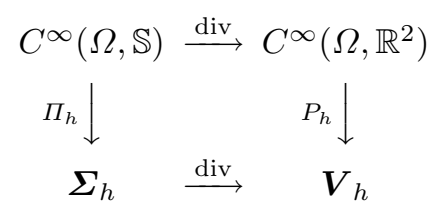

The stability of the mixed method follows from the exactness of 8 , the commutativity of 9 , and the well-posedness of the continuous problem.

Information about the construction of such finite element spaces can be gained by completing the sequence 8 to a sequence analogous to 6 . For this purpose, we set $Q_{h}=\left\{q \in H^{2}(\Omega): J q \in \boldsymbol{\Sigma}_{h}\right\}$. Note $Q_{h}$ is a finite element approximation of $H^{2}(\Omega)$. Moreover, there is a natural interpolation operator $I_{h}: C^{\infty}(\Omega) \mapsto Q_{h}$ so that the following diagram with exact rows commutes:

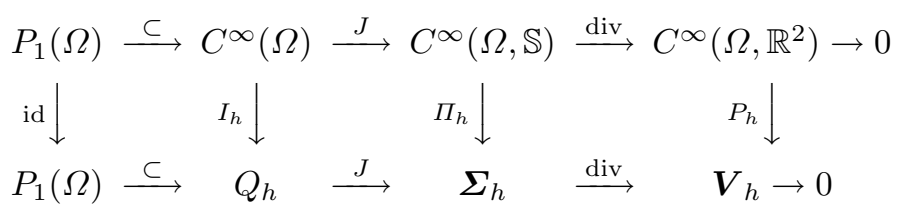

For a description of this construction, see [10]. As discussed there, under quite general conditions, the existence of a stable pair of spaces $\left(\boldsymbol{\Sigma}_{h}, \boldsymbol{V}_{h}\right)$ approximating $H(\operatorname{div}, \Omega ; \mathbb{S}) \times L^{2}\left(\Omega, \mathbb{R}^{2}\right)$, implies the existence of a finite element approximation $Q_{h}$ of $H^{2}(\Omega)$ related to $\boldsymbol{\Sigma}_{h}$ and $\boldsymbol{V}_{h}$ through the diagram above. The fact that the space $Q_{h}$ requires $C^{1}(\Omega)$ finite elements represents a substantial obstruction to the construction of stable mixed elements, and in part accounts for their slow development. In fact, the lowest order element proposed in [10] corresponds to choosing $Q_{h}$ to be the Argyris space of $C^{1}$ piecewise quintic polynomials (the simplest choice). Since $J Q_{h} \subset \boldsymbol{\Sigma}_{h}$, one then sees that $\boldsymbol{\Sigma}_{h}$ must be a piecewise cubic space, and since the Argyris space has second derivative degrees of freedom at the vertices, the degrees of freedom for $\boldsymbol{\Sigma}_{h}$ will include vertex degrees of freedom, not usually expected for subspaces of $H(\operatorname{div} ; \Omega)$.

The family of elements developed in [10] chooses for $k \geq 1$, the local degrees of freedom for $\boldsymbol{\Sigma}_{h}$ to be

$$
\begin{aligned}
\Sigma_{T} & =\mathcal{P}_{k+1}(T, \mathbb{S})+\left\{\tau \in \mathcal{P}_{k+2}(T, \mathbb{S}): \operatorname{div} \tau=0\right\} \\
& =\left\{\tau \in \mathcal{P}_{k+2}(T, \mathbb{S}): \operatorname{div} \tau \in \mathcal{P}_{k}\left(T, \mathbb{R}^{2}\right)\right\}, \quad V_{T}=\mathcal{P}_{k}\left(T, \mathbb{R}^{2}\right)
\end{aligned}
$$


Now $\operatorname{dim} V_{T}=(k+2)(k+1)$ and it is shown in [10] that $\operatorname{dim} \Sigma_{T}=$ $\left(3 k^{2}+17 k+28\right) / 2$ and that a unisolvent set of local degrees of freedom is given by

- the values of 3 components of $\tau(x)$ at each vertex $x$ of $T$ (9 degrees of freedom)

- the values of the moments of degree at most $k$ of the two normal components of $\tau$ on each edge $e$ of $T$ (6k+6 degrees of freedom)

- the value of the moments $\int_{T} \tau: \phi \mathrm{d} x, \phi \in \mathcal{P}_{k}\left(T, \mathbb{R}^{2}\right)+\operatorname{airy}\left(b_{T}^{2} \mathcal{P}_{k-2}(T, \mathbb{R})\right)$.

For this family of elements, it is shown in [10] that

$$
\begin{gathered}
\left\|\sigma-\sigma_{h}\right\|_{0} \leq C h^{r}\|\sigma\|_{r}, \quad 1 \leq r \leq k+2, \\
\left\|\operatorname{div}\left(\sigma-\sigma_{h}\right)\right\|_{0} \leq C h^{r}\|\operatorname{div} \sigma\|_{r}, \quad 0 \leq r \leq k+1, \\
\left\|u-u_{h}\right\|_{0} \leq C h^{r}\|u\|_{r+1}, \quad 1 \leq r \leq k+1 .
\end{gathered}
$$

There is a variant of the lowest degree $(k=1)$ element involving fewer degrees of freedom. In this element, one chooses $V_{T}$ to be the space of infinitesimal rigid motions on $T$, i.e., vector functions of the form $(a-b y, c+b x)$. Then $\Sigma_{T}=\left\{\tau \in \mathcal{P}_{3}(T, \mathbb{S}): \operatorname{div} \tau \in V_{T}\right\}$.

The element diagram for the choice $k=1$ and a simplified element are depicted below.
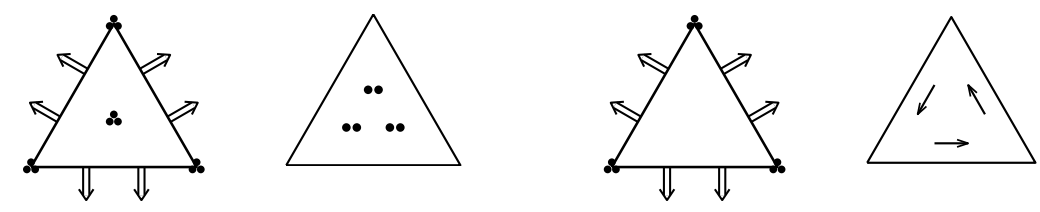

Fig. 1. $k=1$ and simplified Arnold-Winther elements

In [12], the authors obtain simpler elements with fewer degrees of freedom, and also avoid the use of vertex degrees of freedom by developing nonconforming elements. Corresponding to the choice $V_{T}=\mathcal{P}_{1}\left(T, \mathbb{R}^{2}\right)$, one chooses for the stress shape functions

$$
\Sigma_{T}=\left\{\tau \in \mathcal{P}_{2}(T, \mathbb{S}): n \cdot \tau n \in \mathcal{P}_{1}(e, \mathbb{R}), \text { for each edge } e \text { of } T\right\} .
$$

The space $\Sigma_{T}$ has dimension 15, with degrees of freedom given by

- the values of the moments of degree 0 and 1 of the two normal components of $\tau$ on each edge $e$ of $T$ (12 degrees of freedom),

- the value of the three components of the moment of degree 0 of $\tau$ on $T$ (3 degrees of freedom).

Note that this element is a nonconforming approximation of $H(\operatorname{div}, \Omega ; \mathbb{S})$, since although $t \cdot \tau n$ may be quadratic on an edge, only its two lowest order 
moments are determined on each edge. Hence, $\tau n$ may not be continuous across element boundaries. This space may be simplified in a manner similar to the lowest order conforming element, i.e., the displacement space may be chosen to be piecewise rigid motions and the stress space then reduced by requiring that the divergence be a rigid motion on each triangle. The local dimension of the resulting space is 12 and the first two moments of the normal traction on each edge form a unisolvent set of degrees of freedom.
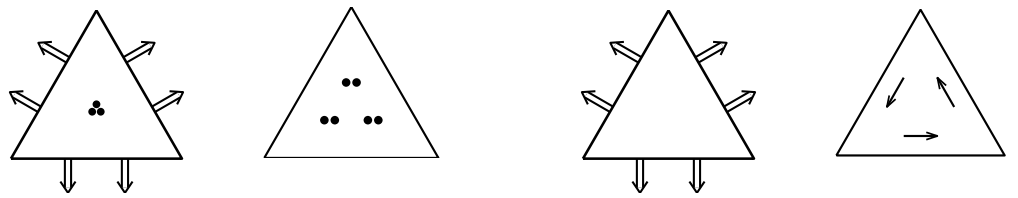

Fig. 2. Two nonconforming Arnold-Winther elements

As noted earlier, for $k=1$, the corresponding space $Q_{h}$ is the Argyris space consisting of $C^{1}$ piecewise quintic polynomials. There is also an analogous relationship for the composite elements discussed earlier. For the element of [24], the space $Q_{h}$ is the Clough-Tocher composite $H^{2}$ element and for the element family of [4], the $Q_{h}$ spaces are the higher order composite elements of $[17]$.
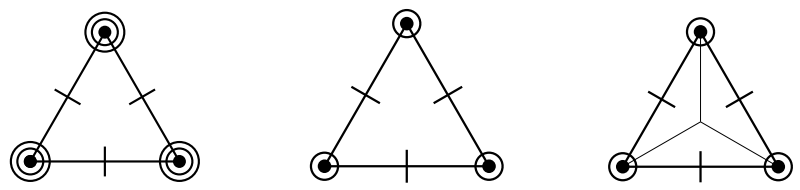

Fig. 3. $Q_{h}$ spaces for $k=1$ conforming element, nonconforming element, and composite element of [24]

The remainder of these notes will be devoted to the development and analysis of mixed finite element methods based on the formulation 3 of the equations of elasticity with weak symmetry. An important advantage of such an approach is that it allows us to approximate the stress matrix by $n$ copies of standard finite element approximations of $\boldsymbol{H}(\operatorname{div}, \Omega)$ used to discretize scalar second order elliptic problems. In fact, to develop our approximation schemes for 3, we will heavily exploit the many close connections between these two problems. Although there is some overhead to the development, much of the structure of these connections is most clearly seen in the language of differential forms. Thus, we devote the next section to a brief overview of the necessary background material. 


\section{Exterior calculus on $\mathbb{R}^{n}$}

To simplify matters, we will consider exterior calculus on $\mathbb{R}^{n}$, and summarize only the specific results we will need.

\subsection{Differential forms}

Suppose that $\Omega$ is an open subset of $\mathbb{R}^{n}$. For $0 \leq k \leq n$, we let $\Lambda^{k}$ denote the space of smooth differential $k$-forms of $\Omega$, i.e., $\Lambda^{k}=\Lambda^{k}(\Omega)=C^{\infty}\left(\Omega ; \operatorname{Alt}^{k} \mathbb{V}\right)$, where $\mathrm{Alt}^{k} \mathbb{V}$ denotes the vector space of alternating $k$-linear maps on $\mathbb{V}$. If $\omega \in \Lambda^{k}(\Omega)$, this means that at each point $x \in \Omega$, there is a map $\omega_{x} \in \operatorname{Alt}^{k} \mathbb{V}$, i.e, $\omega_{x}$ assigns to each $k$-tuple of vectors $v_{1}, \ldots, v_{k}$ of $\mathbb{V}$, a real number $\omega_{x}\left(v_{1}, \ldots, v_{k}\right)$ with the mapping linear in each argument and reversing sign when two arguments are interchanged.

A general element of $\Lambda^{k}(\Omega)$ may be written

$$
\omega_{x}=\sum_{1 \leq \sigma(1)<\cdots<\sigma(k) \leq n} a_{\sigma} \mathrm{d} x_{\sigma(1)} \wedge \cdots \wedge \mathrm{d} x_{\sigma(k)},
$$

where the $a_{\sigma} \in C^{\infty}(\Omega)$. If we allow instead $a_{\sigma} \in C^{p}(\Omega), a_{\sigma} \in L^{2}(\Omega), a_{\sigma} \in$ $H^{s}(\Omega)$, etc., we obtain the spaces $C^{p} \Lambda(\Omega), L^{2} \Lambda(\Omega), H^{s} \Lambda(\Omega)$, etc. Thus, when $n=2$, for $k=0,1,2, \omega \in \Lambda^{k}(\Omega)$ will have the respective forms

$$
w, \quad w_{1} \mathrm{~d} x_{1}+w_{2} \mathrm{~d} x_{2}, \quad w \mathrm{~d} x_{1} \wedge \mathrm{d} x_{2} .
$$

To see the connection between differential forms and scalar and vector-valued functions, we may identify $w \in \Lambda^{0}(\Omega)$ and $w \mathrm{~d} x_{1} \wedge \mathrm{d} x_{2} \in \Lambda^{2}(\Omega)$ with the function $w \in C^{\infty}(\Omega)$ and $w_{1} \mathrm{~d} x_{1}+w_{2} \mathrm{~d} x_{2} \in \Lambda^{1}(\Omega)$ with the vector $\left(w_{1}, w_{2}\right)$ or the vector $\left(-w_{2}, w_{1}\right) \in C^{\infty}\left(\Omega ; \mathbb{R}^{2}\right)$. The associated fields are called proxy fields for the forms.

When $n=3$, for $k=0,1,2,3, \omega \in \Lambda^{k}(\Omega)$ will have the respective forms

$$
\begin{gathered}
w, \quad w_{1} \mathrm{~d} x_{1}+w_{2} \mathrm{~d} x_{2}+w_{3} \mathrm{~d} x_{3}, \quad w_{1} \mathrm{~d} x_{2} \wedge \mathrm{d} x_{3}-w_{2} \mathrm{~d} x_{1} \wedge \mathrm{d} x_{3}+w_{3} \mathrm{~d} x_{1} \wedge \mathrm{d} x_{2}, \\
w \mathrm{~d} x_{1} \wedge \mathrm{d} x_{2} \wedge \mathrm{d} x_{3} .
\end{gathered}
$$

In this case, we may identify $w \in \Lambda^{0}(\Omega)$ and $w \mathrm{~d} x_{1} \wedge \mathrm{d} x_{2} \wedge \mathrm{d} x_{3} \in \Lambda^{3}(\Omega)$ with the function $w \in C^{\infty}(\Omega)$ and $w_{1} \mathrm{~d} x_{1}+w_{2} \mathrm{~d} x_{2}+w_{3} \mathrm{~d} x_{3}$ or $w_{1} \mathrm{~d} x_{2} \wedge \mathrm{d} x_{3}-$ $w_{2} \mathrm{~d} x_{1} \wedge \mathrm{d} x_{3}+w_{3} \mathrm{~d} x_{1} \wedge \mathrm{d} x_{2}$ with the vector $\left(w_{1}, w_{2}, w_{3}\right) \in C^{\infty}\left(\Omega ; \mathbb{R}^{2}\right)$. The correspondences are listed in Table 1 .

To evaluate $\omega_{x}\left(v_{1}, \cdots, v_{k}\right)$, we need a formula for evaluating the $k$-form $\mathrm{d} x_{\sigma(1)} \wedge \cdots \wedge \mathrm{d} x_{\sigma(k)}\left(v_{1}, \ldots, v_{k}\right)$. Rather than presenting the general case, we note that for $v, w, z \in \mathbb{R}^{n}$,

$$
\mathrm{d} x_{i}(v)=v_{i}, \quad \mathrm{~d} x_{i} \wedge \mathrm{d} x_{j}(v, w)=v_{i} w_{j}-v_{j} w_{i},
$$

and for $n=3, \quad \mathrm{~d} x_{1} \wedge \mathrm{d} x_{2} \wedge \mathrm{d} x_{3}(v, w, z)=\operatorname{det}(v|w| z)$. 
Table 1. Correspondence between alternating algebraic forms on $\mathbb{R}^{3}$ and scalars/vectors

\begin{tabular}{|c|c|}
\hline $\operatorname{Alt}^{0} \mathbb{R}^{3}=\mathbb{R}$ & $c \leftrightarrow c$ \\
$\operatorname{Alt}^{1} \mathbb{R}^{3} \cong \mathbb{R}^{3}$ & $u_{1} \mathrm{~d} x_{1}+u_{2} \mathrm{~d} x_{2}+u_{3} \mathrm{~d} x_{3} \leftrightarrow u$ \\
$\operatorname{Alt}^{2} \mathbb{R}^{3} \stackrel{\cong}{\longrightarrow} \mathbb{R}^{3}$ & $u_{3} \mathrm{~d} x_{1} \wedge \mathrm{d} x_{2}-u_{2} \mathrm{~d} x_{1} \wedge \mathrm{d} x_{3}$ \\
& $+u_{1} \mathrm{~d} x_{2} \wedge \mathrm{d} x_{3} \leftrightarrow u$ \\
Alt $^{3} \mathbb{R}^{3} \stackrel{\cong}{\longrightarrow}$ & $c \mathrm{~d} x_{1} \wedge \mathrm{d} x_{2} \wedge \mathrm{d} x_{3} \leftrightarrow c$ \\
\hline
\end{tabular}

For $\omega \in \mathrm{Alt}^{j} V$ and $\eta \in \mathrm{Alt}^{k} V$, the exterior product or wedge product $\omega \wedge$ $\eta \in \mathrm{Alt}^{j+k} V$ is bilinear and associative, and satisfies the anti-commutativity condition

$$
\eta \wedge \omega=(-1)^{j k} \omega \wedge \eta, \quad \omega \in \operatorname{Alt}^{j} V, \eta \in \operatorname{Alt}^{k} V .
$$

Thus, $\mathrm{d} x_{i} \wedge \mathrm{d} x_{j}=-\mathrm{d} x_{j} \wedge \mathrm{d} x_{i}$ and so $\mathrm{d} x_{i} \wedge \mathrm{d} x_{i}=0$.

If $\omega=\sum_{i=1}^{n} w_{i} d x_{i} \in \Lambda^{1}(\Omega)$ and $\eta \in \Lambda^{0}(\Omega)$, then $\omega \wedge \eta$ simply multiplies each of the coefficients $w_{i}$ by $\eta$. If $\eta=\sum_{i=1}^{n} \eta_{i} d x_{i} \in \Lambda^{1}(\Omega)$, then from the bilinearity and antisymmetry, we have

$$
\begin{gathered}
\omega \wedge \eta=w_{1} \eta_{1} \mathrm{~d} x_{1} \wedge \mathrm{d} x_{1}+w_{1} \eta_{2} \mathrm{~d} x_{1} \wedge \mathrm{d} x_{2}+w_{2} \eta_{1} \mathrm{~d} x_{2} \wedge \mathrm{d} x_{1}+w_{2} \eta_{1} \mathrm{~d} x_{2} \wedge \mathrm{d} x_{2} \\
=\left(w_{1} \eta_{2}-w_{2} \eta_{1}\right) \mathrm{d} x_{1} \wedge \mathrm{d} x_{2}, \quad n=2, \\
\omega \wedge \eta=\left(w_{1} \eta_{2}-w_{2} \eta_{1}\right) \mathrm{d} x_{1} \wedge \mathrm{d} x_{2}+\left(w_{1} \eta_{3}-w_{3} \eta_{1}\right) \mathrm{d} x_{1} \wedge \mathrm{d} x_{3} \\
+\left(w_{2} \eta_{3}-w_{3} \eta_{2}\right) \mathrm{d} x_{2} \wedge \mathrm{d} x_{3}, \quad n=3 .
\end{gathered}
$$

Finally, if $\eta \in \Lambda^{2}(\Omega)=\eta_{1} \mathrm{~d} x_{2} \wedge \mathrm{d} x_{3}-\eta_{2} \mathrm{~d} x_{1} \wedge \mathrm{d} x_{3}+\eta_{3} \mathrm{~d} x_{1} \wedge \mathrm{d} x_{2}$, then

$$
\omega \wedge \eta=\left(w_{1} \eta_{1}+w_{2} \eta_{2}+w_{3} \eta_{3}\right) \mathrm{d} x_{1} \wedge \mathrm{d} x_{2} \wedge \mathrm{d} x_{3} .
$$

One can give a general formula for the wedge product, which we omit here.

If $\omega_{x}$ and $\eta_{x} \in \Lambda^{k}(\Omega)$ are given by

$\sum_{1 \leq \sigma(1)<\cdots<\sigma(k) \leq n} a_{\sigma} \mathrm{d} x_{\sigma(1)} \wedge \cdots \wedge \mathrm{d} x_{\sigma(k)}, \sum_{1 \leq \sigma(1)<\cdots<\sigma(k) \leq n} b_{\sigma} \mathrm{d} x_{\sigma(1)} \wedge \cdots \wedge \mathrm{d} x_{\sigma(k)}$,

respectively, we can define the inner products

$$
\left\langle\omega_{x}, \eta_{x}\right\rangle=\sum_{1 \leq \sigma(1)<\cdots<\sigma(k) \leq n} a_{\sigma} b_{\sigma}, \quad\langle\omega, \eta\rangle=\int_{\Omega}\left\langle\omega_{x}, \eta_{x}\right\rangle \mathrm{d} x_{1} \wedge \cdots \wedge \mathrm{d} x_{n},
$$

where $\mathrm{d} x_{1} \wedge \cdots \wedge \mathrm{d} x_{n}$ is the volume form.

A key object in our presentation is the exterior derivative $\mathrm{d}=\mathrm{d}_{k}$ : $\Lambda^{k}(\Omega) \rightarrow \Lambda^{k+1}(\Omega)$, defined by

$$
\mathrm{d} \sum a_{\sigma} \mathrm{d} x_{\sigma(1)} \wedge \cdots \wedge \mathrm{d} x_{\sigma(k)}=\sum_{\sigma} \sum_{i=1}^{n} \frac{\partial a_{\sigma}}{\partial x_{i}} \mathrm{~d} x_{i} \wedge \mathrm{d} x_{\sigma(1)} \wedge \cdots \wedge \mathrm{d} x_{\sigma(k)} .
$$


Table 2. Correspondences between differential forms $\omega$ on $\Omega \subset \mathbb{R}^{3}$ and scalar/vector fields $w$ on $\Omega$.

\begin{tabular}{|c|ccc|}
\hline$k$ & $\Lambda^{k}(\Omega)$ & $H \Lambda^{k}(\Omega)$ & $\mathrm{d} \omega$ \\
\hline 0 & $C^{\infty}(\Omega)$ & $H^{1}(\Omega)$ & $\operatorname{grad} w$ \\
1 & $C^{\infty}\left(\Omega ; \mathbb{R}^{3}\right)$ & $H\left(\mathbf{c u r l}, \Omega ; \mathbb{R}^{3}\right)$ & $\operatorname{curl} w$ \\
2 & $C^{\infty}\left(\Omega ; \mathbb{R}^{3}\right)$ & $H\left(\operatorname{div}, \Omega ; \mathbb{R}^{3}\right)$ & $\operatorname{div} w$ \\
3 & $C^{\infty}(\Omega)$ & $L^{2}(\Omega)$ & 0 \\
\hline
\end{tabular}

As we shall see below, the exterior derivative operator d corresponds to the standard differential operators grad, curl, div, and rot.

When $n=2$, if $\omega \in \Lambda^{0}(\Omega)$, then $\mathrm{d}_{0} \omega=\partial w / \partial x_{1} \mathrm{~d} x_{1}+\partial w / \partial x_{2} \mathrm{~d} x_{2} \in \Lambda^{1}(\Omega)$. Identifying $\partial w / \partial x_{1} \mathrm{~d} x_{1}+\partial w / \partial x_{2} \mathrm{~d} x_{2}$ with the vector $\left(\partial w / \partial x_{1}, \partial w / \partial x_{2}\right), \mathrm{d}_{0}$ corresponds to grad. If instead, we identify $\partial w / \partial x_{1} \mathrm{~d} x_{1}+\partial w / \partial x_{2} \mathrm{~d} x_{2}$ with the vector $\left(-\partial w / \partial x_{2}, \partial w / \partial x_{1}\right)$, then $\mathrm{d}_{0}$ corresponds to curl.

If $\mu=w_{1} \mathrm{~d} x_{1}+w_{2} \mathrm{~d} x_{2} \in \Lambda^{1}(\Omega)$, then $\mathrm{d}_{1} \mu=\left(\partial w_{2} / \partial x_{1}-\partial w_{1} / \partial x_{2}\right) \mathrm{d} x_{1} \wedge$ $\mathrm{d} x_{2} \in \Lambda^{2}(\Omega)$. If we identify $w_{1} \mathrm{~d} x_{1}+w_{2} \mathrm{~d} x_{2}$ with the vector $\left(w_{1}, w_{2}\right)$, then $\mathrm{d}_{1}$ corresponds to rot. If instead, we identify $w_{1} \mathrm{~d} x_{1}+w_{2} \mathrm{~d} x_{2}$ with the vector $\left(-w_{2}, w_{1}\right)$, then $\mathrm{d}_{1}$ corresponds to - div.

When $n=3$, if $\omega \in \Lambda^{0}(\Omega)$, then $\mathrm{d}_{0} \omega=\partial w / \partial x_{1} \mathrm{~d} x_{1}+\partial w / \partial x_{2} \mathrm{~d} x_{2}+$ $\partial w / \partial x_{3} \mathrm{~d} x_{3} \in \Lambda^{1}(\Omega)$. Identifying $\partial w / \partial x_{1} \mathrm{~d} x_{1}+\partial w / \partial x_{2} \mathrm{~d} x_{2}+\partial w / \partial x_{3} \mathrm{~d} x_{3}$ with $\left(\partial w / \partial x_{1}, \partial w / \partial x_{2}, \partial w / \partial x_{3}\right), \mathrm{d}_{0}$ corresponds to grad. If $\mu=w_{1} \mathrm{~d} x_{1}+w_{2} \mathrm{~d} x_{2}+$ $w_{3} \mathrm{~d} x_{3} \in \Lambda^{1}(\Omega)$, then $\mathrm{d}_{1} \mu=\left(\partial w_{3} / \partial x_{2}-\partial w_{2} / \partial x_{3}\right) \mathrm{d} x_{2} \wedge \mathrm{d} x_{3}-\left(\partial w_{1} / \partial x_{3}-\right.$ $\left.\partial w_{3} / \partial x_{1}\right) \mathrm{d} x_{1} \wedge \mathrm{d} x_{3}+\left(\partial w_{2} / \partial x_{1}-\partial w_{1} / \partial x_{2}\right) \mathrm{d} x_{1} \wedge \mathrm{d} x_{2} \in \Lambda^{2}(\Omega)$. Identifying $w_{1} \mathrm{~d} x_{1}+w_{2} \mathrm{~d} x_{2}+w_{3} \mathrm{~d} x_{3}$ with the vector $\left(w_{1}, w_{2}, w_{3}\right), \mathrm{d}_{1}$ corresponds to curl. Finally, if $\mu=w_{1} \mathrm{~d} x_{2} \wedge \mathrm{d} x_{3}-w_{2} \mathrm{~d} x_{1} \wedge \mathrm{d} x_{3}+w_{3} \mathrm{~d} x_{1} \wedge \mathrm{d} x_{2} \in \Lambda^{2}(\Omega)$, then $\mathrm{d}_{2} \mu=\left(\partial w_{1} / \partial x_{1}+\partial w_{2} / \partial x_{2}+\partial w_{3} / \partial x_{3}\right) \mathrm{d} x_{1} \wedge \mathrm{d} x_{2} \wedge \mathrm{d} x_{3} \in \Lambda^{3}(\Omega)$. Identifying $\mu$ with $\left(w_{1}, w_{2}, w_{3}\right), \mathrm{d}_{2}$ corresponds to div. Table 2 summarizes correspondences between differential forms and their proxy fields in the case $\Omega \subset \mathbb{R}^{3}$.

An important role in our analysis is played by the de Rham sequence, the sequence of spaces and mappings given in the notation of differential forms by:

$$
\mathbb{R} \stackrel{\subset}{\longrightarrow} \Lambda^{0}(\Omega) \stackrel{\mathrm{d}_{0}}{\longrightarrow} \Lambda^{1}(\Omega) \stackrel{\mathrm{d}_{1}}{\longrightarrow} \cdots \stackrel{\mathrm{d}_{n-1}}{\longrightarrow} \Lambda^{n}(\Omega) \rightarrow 0 .
$$

By introducing proxy fields and the usual differential operators, the de Rham complex (and its $L^{2}$ version) take the following forms. For $\Omega \subset \mathbb{R}^{3}$,

$$
\begin{gathered}
\mathbb{R} \stackrel{\subseteq}{\hookrightarrow} C^{\infty}(\Omega) \stackrel{\text { grad }}{\longrightarrow} C^{\infty}\left(\Omega ; \mathbb{R}^{3}\right) \stackrel{\text { curl }}{\longrightarrow} C^{\infty}\left(\Omega ; \mathbb{R}^{3}\right) \stackrel{\text { div }}{\longrightarrow} C^{\infty}(\Omega) \rightarrow 0, \\
\mathbb{R} \stackrel{\subseteq}{\subseteq} H^{1}(\Omega) \stackrel{\text { grad }}{\longrightarrow} H\left(\text { curl, } \Omega ; \mathbb{R}^{3}\right) \stackrel{\text { curl }}{\longrightarrow} H\left(\operatorname{div}, \Omega ; \mathbb{R}^{3}\right) \stackrel{\text { div }}{\longrightarrow} L^{2}(\Omega) \rightarrow 0 .
\end{gathered}
$$

For $\Omega \subset \mathbb{R}^{2}$, the de Rham complex becomes

$$
\mathbb{R} \stackrel{\subset}{\longrightarrow} C^{\infty}(\Omega) \stackrel{\text { grad }}{\longrightarrow} C^{\infty}\left(\Omega ; \mathbb{R}^{2}\right) \stackrel{\text { rot }}{\longrightarrow} C^{\infty}(\Omega) \rightarrow 0
$$

or 


$$
\mathbb{R} \stackrel{\subset}{\longrightarrow} C^{\infty}(\Omega) \stackrel{\text { curl }}{\longrightarrow} C^{\infty}\left(\Omega ; \mathbb{R}^{2}\right) \stackrel{\text { div }}{\longrightarrow} C^{\infty}(\Omega) \rightarrow 0,
$$

depending on whether we identify $w_{1} \mathrm{~d} x_{1}+w_{2} \mathrm{~d} x_{2} \in \Lambda^{1}(\Omega)$ with the vector $\left(w_{1}, w_{2}\right)$ or the vector $\left(-w_{2}, w_{1}\right)$. There are also analogous $L^{2}$ complexes.

\section{Basic finite element spaces and their properties}

We now turn to the definition of the finite element spaces we shall use in our approximation schemes and their properties. For this we follow the approach developed in [9]. We begin by defining $\mathcal{P}_{r}$ as the space of polynomials in $n$ variables of degree at most $r$ and $\mathcal{P}_{r} \Lambda^{k}$ as the space of differential $k$-forms with coefficients belonging to $\mathcal{P}_{r}$. Let $\mathcal{T}_{h}$ be a triangulation of $\Omega$ by $n+1$ simplices $T$ and set

$$
\begin{aligned}
\mathcal{P}_{r} \Lambda^{k}\left(\mathcal{T}_{h}\right) & =\left\{\omega \in H \Lambda^{k}(\Omega): \omega_{T} \in \mathcal{P}_{r} \Lambda^{k}(T) \forall T \in \mathcal{T}_{h}\right\}, \quad r \geq 0 \\
\mathcal{P}_{r}^{-} \Lambda^{k}\left(\mathcal{T}_{h}\right) & =\left\{\omega \in H \Lambda^{k}(\Omega): \omega_{T} \in \mathcal{P}_{r}^{-} \Lambda^{k}(T) \forall T \in \mathcal{T}_{h}\right\}, \quad r \geq 1,
\end{aligned}
$$

where $\mathcal{P}_{r}^{-} \Lambda^{k}(T):=\mathcal{P}_{r-1} \Lambda^{k}(T)+\kappa \mathcal{P}_{r-1} \Lambda^{k+1}(T)$ and $\kappa=\kappa_{k+1}: \Lambda^{k+1}(T) \rightarrow$ $\Lambda^{k}(T)$ is the Koszul differential defined for $\omega=\sum_{\sigma} a_{\sigma} \mathrm{d} x_{\sigma(1)} \wedge \cdots \wedge \mathrm{d} x_{\sigma(k+1)} \in$ $\Lambda^{k+1}$ by

$$
\kappa \omega=\sum_{\sigma} \sum_{i=1}^{k+1}(-1)^{i+1} a_{\sigma} x_{\sigma(i)} \mathrm{d} x_{\sigma(1)} \wedge \cdots \wedge \hat{\mathrm{d}} x_{\sigma(i)} \wedge \cdots \mathrm{d} x_{\sigma(k+1)},
$$

where the notation $\hat{\mathrm{d}} x_{\sigma(i)}$ means that the term is omitted in the sum. Note that $\kappa_{k} \kappa_{k+1}=0$, and one can show that the Koszul complex

$$
0 \rightarrow \mathcal{P}_{r-n} \Lambda^{n}(\Omega) \stackrel{\kappa_{n}}{\longrightarrow} \mathcal{P}_{r-n+1} \Lambda^{n-1}(\Omega) \stackrel{\kappa_{n-1}}{\longrightarrow} \cdots \stackrel{\kappa_{1}}{\longrightarrow} \mathcal{P}_{r} \Lambda^{0}(\Omega) \rightarrow 0,
$$

is exact. For $\Omega \subset \mathbb{R}^{3}$, this complex becomes

$$
0 \rightarrow \mathcal{P}_{r-3}(\Omega) \stackrel{x}{\rightarrow} \mathcal{P}_{r-2}\left(\Omega ; \mathbb{R}^{3}\right) \stackrel{\times x}{\longrightarrow} \mathcal{P}_{r-1}\left(\Omega ; \mathbb{R}^{3}\right) \stackrel{\cdot x}{\longrightarrow} \mathcal{P}_{r}(\Omega) \rightarrow 0 .
$$

Comparing to the corresponding polynomial de Rham complex

$$
0 \rightarrow \mathcal{P}_{r}(\Omega) \stackrel{\text { grad }}{\longrightarrow} \mathcal{P}_{r-1}\left(\Omega ; \mathbb{R}^{3}\right) \stackrel{\text { curl }}{\longrightarrow} \mathcal{P}_{r-2}\left(\Omega ; \mathbb{R}^{3}\right) \stackrel{\text { div }}{\longrightarrow} \mathcal{P}_{r-3}(\Omega) \rightarrow 0,
$$

we see that the Koszul differential increases polynomial degree and decreases the order of the differential form, while exterior differentiation does exactly the opposite.

We note that $\mathcal{P}_{r} \Lambda^{0}\left(\mathcal{T}_{h}\right)=\mathcal{P}_{r}^{-} \Lambda^{0}\left(\mathcal{T}_{h}\right), r \geq 1$ and $\mathcal{P}_{r} \Lambda^{n}\left(\mathcal{T}_{h}\right)=\mathcal{P}_{r+1}^{-} \Lambda^{n}\left(\mathcal{T}_{h}\right)$, $r \geq 0$. Using proxy fields, we can identify these spaces of finite element differential forms with finite element spaces of scalar and vector functions. In Tables 3 and 4, we summarize the correspondences between spaces of finite 
Table 3. Correspondences between finite element differential forms and the classical finite element spaces for $n=2$.

\begin{tabular}{|c|c|l|}
\hline$k$ & $\Lambda_{h}^{k}(\Omega)$ & \multicolumn{1}{|c|}{ Classical finite element space } \\
\hline 0 & $\mathcal{P}_{r} \Lambda^{0}\left(\mathcal{T}_{h}\right)$ & Lagrange elements of degree $\leq r$ \\
1 & $\mathcal{P}_{r} \Lambda^{1}\left(\mathcal{T}_{h}\right)$ & Brezzi-Douglas-Marini $H($ div) elements of degree $\leq r$ \\
2 & $\mathcal{P}_{r} \Lambda^{2}\left(\mathcal{T}_{h}\right)$ & discontinuous elements of degree $\leq r$ \\
\hline 0 & $\mathcal{P}_{r}^{-} \Lambda^{0}\left(\mathcal{T}_{h}\right)$ & Lagrange elements of degree $\leq r$ \\
1 & $\mathcal{P}_{r}^{-} \Lambda^{1}\left(\mathcal{T}_{h}\right)$ & Raviart-Thomas $H($ div) elements of order $r-1$ \\
2 & $\mathcal{P}_{r}^{-} \Lambda^{2}\left(\mathcal{T}_{h}\right)$ & discontinuous elements of degree $\leq r-1$ \\
\hline
\end{tabular}

Table 4. Correspondences between finite element differential forms and the classical finite element spaces for $n=3$.

\begin{tabular}{|c|c|l|}
\hline$k$ & $\Lambda_{h}^{k}(\Omega)$ & \multicolumn{1}{|c|}{ Classical finite element space } \\
\hline 0 & $\mathcal{P}_{r} \Lambda^{0}\left(\mathcal{T}_{h}\right)$ & Lagrange elements of degree $\leq r$ \\
1 & $\mathcal{P}_{r} \Lambda^{1}\left(\mathcal{T}_{h}\right)$ & Nédélec 2nd-kind $H($ curl $)$ elements of degree $\leq r$ \\
2 & $\mathcal{P}_{r} \Lambda^{2}\left(\mathcal{T}_{h}\right)$ & Nédélec 2nd-kind $H($ div) elements of degree $\leq r$ \\
3 & $\mathcal{P}_{r} \Lambda^{3}\left(\mathcal{T}_{h}\right)$ & discontinuous elements of degree $\leq r$ \\
\hline 0 & $\mathcal{P}_{r}^{-} \Lambda^{0}\left(\mathcal{T}_{h}\right)$ & Lagrange elements of degree $\leq r$ \\
1 & $\mathcal{P}_{r}^{-} \Lambda^{1}\left(\mathcal{T}_{h}\right)$ & Nédélec 1st-kind $H($ curl $)$ elements of order $r-1$ \\
2 & $\mathcal{P}_{r}^{-} \Lambda^{2}\left(\mathcal{T}_{h}\right)$ & Nédélec 1st-kind $H($ div) elements of order $r-1$ \\
3 & $\mathcal{P}_{r}^{-} \Lambda^{3}\left(\mathcal{T}_{h}\right)$ & discontinuous elements of degree $\leq r-1$ \\
\hline
\end{tabular}

element differential forms and classical finite element spaces in two and three dimensions.

Degrees of freedom for these spaces are given as follows. For the space $\mathcal{P}_{r} \Lambda^{k}(\mathcal{T})$, we use

$$
\int_{f} \operatorname{Tr}_{f} \omega \wedge \nu, \quad \nu \in \mathcal{P}_{r-j+k}^{-} \Lambda^{j-k}(f), \quad f \in \Delta_{j}(\mathcal{T}),
$$

for $k \leq j \leq \min (n, r+k-1)$, where $\operatorname{Tr}_{f} \omega$ denotes the trace of $\omega$ on the face $f$ and $\Delta_{j}(\mathcal{T})$ is the set of all $j$-dimensional subsimplices generated by $\mathcal{T}_{h}$. For example, when $n=3, \Delta_{j}(\mathcal{T})$ is the set of vertices, edges, faces, or tetrahedra in the mesh $\mathcal{T}_{h}$ for $j=0,1,2,3$. In this case, when $j=0$, i.e., $f$ is a vertex, $\int_{f} \operatorname{Tr}_{f} \omega$ means $w(f)$, where $w$ is the function associated with $\omega \in \Lambda^{0}(\Omega)$. When $j=1$, i.e., $f$ is an edge of a tetrahedron, $\int_{f} \operatorname{Tr}_{f} \omega=\int_{f} w \cdot t d \mu$, where $w$ is the vector associated to $\omega \in \Lambda^{1}(\Omega)$ and $t$ is the unit tangent vector to $f$. When $j=2$, i.e., $f$ is a face of a tetrahedron, $\int_{f} \operatorname{Tr}_{f} \omega=\int_{f} w \cdot n d \mu$, where $w$ is the vector associated to $\omega \in \Lambda^{2}(\Omega)$ and $n$ is the unit outward normal to $f$. Finally, when $j=3$, i.e., $f$ is a tetrahedron, $\int_{f} \operatorname{Tr}_{f} \omega=\int_{f} w d \mu$, where $w$ is the function associated to $\omega \in \Lambda^{3}(\Omega)$. 
Analogously, the degrees of freedom for the space $\mathcal{P}_{r}^{-} \Lambda^{k}(\mathcal{T})$ are given by

$$
\int_{f} \operatorname{Tr}_{f} \omega \wedge \nu, \quad \nu \in \mathcal{P}_{r-j+k-1} \Lambda^{j-k}(f), \quad f \in \Delta_{j}(\mathcal{T}),
$$

for $k \leq j \leq \min (n, r+k-1)$. Note the key property that the degrees of freedom for each space are defined in terms of wedge products with elements of the other space.

An important property of these finite element spaces is that they form discrete de Rham sequences. In fact, as shown in [9], in $n$ dimensions, there are exactly $2^{n-1}$ distinct sequences. When $n=2$ and $r \geq 0$, these are

$$
\begin{aligned}
& 0 \rightarrow \mathcal{P}_{r+2} \Lambda^{0}\left(\mathcal{T}_{h}\right) \stackrel{\mathrm{d}_{0}}{\longrightarrow} \mathcal{P}_{r+1} \Lambda^{1}\left(\mathcal{T}_{h}\right) \stackrel{\mathrm{d}_{1}}{\longrightarrow} \mathcal{P}_{r} \Lambda^{2}\left(\mathcal{T}_{h}\right) \rightarrow 0, \\
& 0 \rightarrow \mathcal{P}_{r+1} \Lambda^{0}\left(\mathcal{T}_{h}\right) \stackrel{\mathrm{d}_{0}}{\longrightarrow} \mathcal{P}_{r+1}^{-} \Lambda^{1}\left(\mathcal{T}_{h}\right) \stackrel{\mathrm{d}_{1}}{\longrightarrow} \mathcal{P}_{r} \Lambda^{2}\left(\mathcal{T}_{h}\right) \rightarrow 0 .
\end{aligned}
$$

When $n=3$ and $r \geq 0$, we have the four sequences

$$
\begin{aligned}
& 0 \rightarrow \mathcal{P}_{r+3} \Lambda^{0}\left(\mathcal{T}_{h}\right) \stackrel{\mathrm{d}_{0}}{\longrightarrow} \mathcal{P}_{r+2} \Lambda^{1}\left(\mathcal{T}_{h}\right) \stackrel{\mathrm{d}_{1}}{\longrightarrow} \mathcal{P}_{r+1} \Lambda^{2}\left(\mathcal{T}_{h}\right) \stackrel{\mathrm{d}_{2}}{\longrightarrow} \mathcal{P}_{r} \Lambda^{3}\left(\mathcal{T}_{h}\right) \rightarrow 0, \\
& 0 \rightarrow \mathcal{P}_{r+2} \Lambda^{0}\left(\mathcal{T}_{h}\right) \stackrel{\mathrm{d}_{0}}{\longrightarrow} \mathcal{P}_{r+1} \Lambda^{1}\left(\mathcal{T}_{h}\right) \stackrel{\mathrm{d}_{1}}{\longrightarrow} \mathcal{P}_{r+1}^{-} \Lambda^{2}\left(\mathcal{T}_{h}\right) \stackrel{\mathrm{d}_{2}}{\longrightarrow} \mathcal{P}_{r} \Lambda^{3}\left(\mathcal{T}_{h}\right) \rightarrow 0, \\
& 0 \rightarrow \mathcal{P}_{r+2} \Lambda^{0}\left(\mathcal{T}_{h}\right) \stackrel{\mathrm{d}_{0}}{\longrightarrow} \mathcal{P}_{r+2}^{-} \Lambda^{1}\left(\mathcal{T}_{h}\right) \stackrel{\mathrm{d}_{1}}{\longrightarrow} \mathcal{P}_{r+1} \Lambda^{2}\left(\mathcal{T}_{h}\right) \stackrel{\mathrm{d}_{2}}{\longrightarrow} \mathcal{P}_{r} \Lambda^{3}\left(\mathcal{T}_{h}\right) \rightarrow 0, \\
& 0 \rightarrow \mathcal{P}_{r+1} \Lambda^{0}\left(\mathcal{T}_{h}\right) \stackrel{\mathrm{d}_{0}}{\longrightarrow} \mathcal{P}_{r+1}^{-} \Lambda^{1}\left(\mathcal{T}_{h}\right) \stackrel{\mathrm{d}_{1}}{\longrightarrow} \mathcal{P}_{r+1}^{-} \Lambda^{2}\left(\mathcal{T}_{h}\right) \stackrel{\mathrm{d}_{2}}{\longrightarrow} \mathcal{P}_{r} \Lambda^{3}\left(\mathcal{T}_{h}\right) \rightarrow 0 .
\end{aligned}
$$

The first and last of these are exact sequences involving only the $\mathcal{P}_{r} \Lambda^{k}\left(\mathcal{T}_{h}\right)$ or $\mathcal{P}_{r}^{-} \Lambda^{k}\left(\mathcal{T}_{h}\right)$ spaces alone, while the middle two mix the two spaces. As we shall see, to obtain mixed finite element methods for elasticity when $n=3$, it is one of these middle sequences that will play a key role.

To each of the spaces $\mathcal{P}_{r} \Lambda^{k}\left(\mathcal{T}_{h}\right)$, we may associate a canonical projection operator $\Pi\left(=\Pi_{\mathcal{T}_{h}}\right): C^{0} \Lambda^{k}(\Omega) \rightarrow \mathcal{P}_{r} \Lambda^{k}\left(\mathcal{T}_{h}\right)$ defined by the equations:

$$
\int_{f} \operatorname{Tr}_{f} \Pi \omega \wedge \nu=\int_{f} \operatorname{Tr}_{f} \omega \wedge \nu, \quad \nu \in \mathcal{P}_{r-j+k}^{-} \Lambda^{j-k}(f), \quad f \in \Delta_{j}(\mathcal{T}),
$$

for $k \leq j \leq \min (n, r+k-1)$. Similarly, to each of the spaces $\mathcal{P}_{r}^{-} \Lambda^{k}\left(\mathcal{T}_{h}\right)$, we may associate a canonical projection operator $\Pi\left(=\Pi_{\mathcal{T}_{h}}\right): C^{0} \Lambda^{k}(\Omega) \rightarrow \mathcal{P}_{r}^{-} \Lambda^{k}\left(\mathcal{T}_{h}\right)$ defined by the equations

$$
\int_{f} \operatorname{Tr}_{f} \Pi \omega \wedge \nu=\int_{f} \operatorname{Tr}_{f} \omega \wedge \nu, \quad \nu \in \mathcal{P}_{r-j+k-1} \Lambda^{j-k}(f), \quad f \in \Delta_{j}(\mathcal{T}),
$$

for $k \leq j \leq \min (n, r+k-1)$. A key property of these projection operators is that they commute with the exterior derivative, i.e., the following four diagrams commute. 


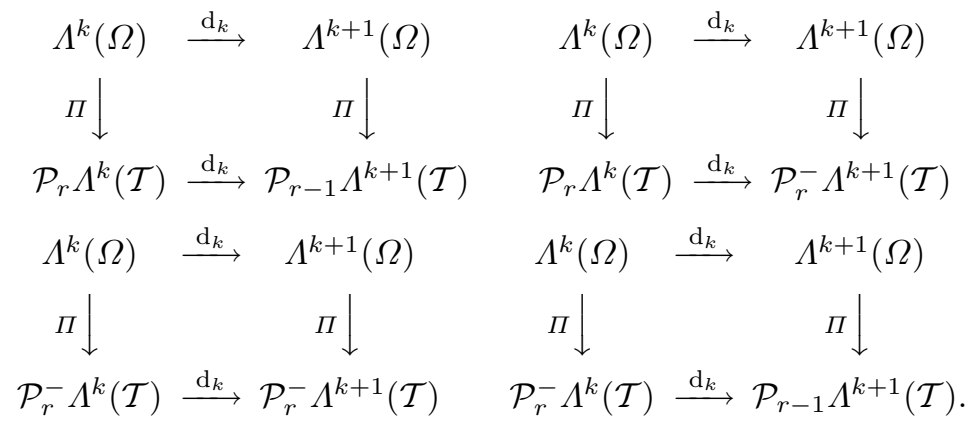

These commuting diagrams will also play an essential role in the construction of stable mixed finite element approximation schemes for the equations of elasticity.

\subsection{Differential forms with values in a vector space}

To study the equations of linear elasticity in the language of differential forms, we will need to use differential forms with values in a vector space. Let $V$ and $W$ be finite dimensional vector spaces. We then define the space $\Lambda^{k}(V ; W)$ of differential forms on $V$ with values in $W$. The two examples we have in mind are when $V=\mathbb{V}=\mathbb{R}^{n}$ and $W=\mathbb{V}$ or $W=\mathbb{K}$, the set of anti-symmetric matrices. When $n=2, \omega \in \Lambda^{k}(\mathbb{V} ; \mathbb{V}), k=0,1,2$ will have the respective forms

$$
\left(\begin{array}{l}
w_{1} \\
w_{2}
\end{array}\right), \quad\left(\begin{array}{l}
w_{11} \\
w_{21}
\end{array}\right) \mathrm{d} x_{1}+\left(\begin{array}{c}
w_{12} \\
w_{22}
\end{array}\right) \mathrm{d} x_{2}, \quad\left(\begin{array}{c}
w_{1} \\
w_{2}
\end{array}\right) \mathrm{d} x_{1} \wedge \mathrm{d} x_{2},
$$

while $\omega \in \Lambda^{k}(\mathbb{V} ; \mathbb{K})$ will have the respective forms

$w \boldsymbol{\chi}, \quad w_{1} \chi \mathrm{d} x_{1}+w_{2} \chi \mathrm{d} x_{2}, \quad w \boldsymbol{x} x_{1} \wedge \mathrm{d} x_{2}, \quad$ where $\quad \boldsymbol{\chi}=\left(\begin{array}{cc}0 & -1 \\ 1 & 0\end{array}\right)$.

Recalling that the 1 -form $w_{1} \mathrm{~d} x_{1}+w_{2} \mathrm{~d} x_{2}$ can be identified either with the vector $\left(w_{1}, w_{2}\right)$ or the vector $\left(-w_{2}, w_{1}\right)$, we will have the analogous possibilities in the case of vector or matrix-valued forms. Since we will be interested in de Rham sequences involving the operator div, we choose the second identification. Hence, $\left(\begin{array}{l}w_{11} \\ w_{21}\end{array}\right) \mathrm{d} x_{1}+\left(\begin{array}{l}w_{12} \\ w_{22}\end{array}\right) \mathrm{d} x_{2} \in \Lambda^{1}(\mathbb{V} ; \mathbb{V})$ will be identified with the matrix

$$
\left(\begin{array}{ll}
W_{11} & W_{12} \\
W_{21} & W_{22}
\end{array}\right)=\left(\begin{array}{ll}
-w_{12} & w_{11} \\
-w_{22} & w_{21}
\end{array}\right)
$$

and $w_{1} \chi \mathrm{d} x_{1}+w_{2} \chi \mathrm{d} x_{2} \in \Lambda^{1}(\mathbb{V} ; \mathbb{K})$ with the vector $\left(-w_{2}, w_{1}\right)$. When $n=3$, $\omega \in \Lambda^{k}(\mathbb{V} ; \mathbb{V})$ will have the respective forms 


$$
\begin{gathered}
\left(\begin{array}{l}
w_{1} \\
w_{2} \\
w_{3}
\end{array}\right), \quad\left(\begin{array}{l}
w_{11} \\
w_{21} \\
w_{31}
\end{array}\right) \mathrm{d} x_{1}+\left(\begin{array}{l}
w_{12} \\
w_{22} \\
w_{32}
\end{array}\right) \mathrm{d} x_{2}+\left(\begin{array}{l}
w_{13} \\
w_{23} \\
w_{33}
\end{array}\right) \mathrm{d} x_{3}, \\
\left(\begin{array}{l}
w_{11} \\
w_{21} \\
w_{31}
\end{array}\right) \mathrm{d} x_{2} \wedge \mathrm{d} x_{3}-\left(\begin{array}{l}
w_{12} \\
w_{22} \\
w_{32}
\end{array}\right) \mathrm{d} x_{1} \wedge \mathrm{d} x_{3}+\left(\begin{array}{l}
w_{13} \\
w_{23} \\
w_{33}
\end{array}\right) \mathrm{d} x_{1} \wedge \mathrm{d} x_{2}, \\
\left(\begin{array}{l}
w_{1} \\
w_{2} \\
w_{3}
\end{array}\right) \mathrm{d} x_{1} \wedge \mathrm{d} x_{2} \wedge \mathrm{d} x_{3} .
\end{gathered}
$$

Hence, $\Lambda^{0}(\mathbb{V} ; \mathbb{V})$ and $\Lambda^{3}(\mathbb{V} ; \mathbb{V})$ have obvious identifications with the space of 3 dimensional vectors and $\Lambda^{1}(\mathbb{V} ; \mathbb{V})$ and $\Lambda^{2}(\mathbb{V} ; \mathbb{V})$ have obvious identifications with the space of $3 \times 3$ matrices (i.e, $W_{i j}=w_{i j}$ in both cases). In fact, in treating the equations of elasticity on a domain $\Omega \subset \mathbb{R}^{n}$, we shall represent the stress as an element of $\Lambda^{n-1}(\Omega, \mathbb{V})$. To describe $\Lambda^{k}(\mathbb{V} ; \mathbb{K})$, it will be convenient to introduce the operator Skw taking a 3 -vector to a skew-symmetric matrix. i.e.,

$$
\operatorname{Skw}\left(w_{1}, w_{2}, w_{3}\right)=\left(\begin{array}{ccc}
0 & -w_{3} & w_{2} \\
w_{3} & 0 & -w_{1} \\
-w_{2} & w_{1} & 0
\end{array}\right) .
$$

Then $\omega \in \Lambda^{k}(\mathbb{V} ; \mathbb{K})$ will have the respective forms

$$
\begin{gathered}
\operatorname{Skw}\left(w_{1}, w_{2}, w_{3}\right), \\
\operatorname{Skw}\left(w_{11}, w_{21}, w_{31}\right) \mathrm{d} x_{1}+\operatorname{Skw}\left(w_{12}, w_{22}, w_{32}\right) \mathrm{d} x_{2}+\operatorname{Skw}\left(w_{13}, w_{23}, w_{33}\right) \mathrm{d} x_{3}, \\
\operatorname{Skw}\left(w_{11}, w_{21}, w_{31}\right) \mathrm{d} x_{2} \wedge \mathrm{d} x_{3}-\operatorname{Skw}\left(w_{12}, w_{22}, w_{32}\right) \mathrm{d} x_{1} \wedge \mathrm{d} x_{3} \\
+\operatorname{Skw}\left(w_{13}, w_{23}, w_{33}\right) \mathrm{d} x_{1} \wedge \mathrm{d} x_{2}, \\
\operatorname{Skw}\left(w_{1}, w_{2}, w_{3}\right) \mathrm{d} x_{1} \wedge \mathrm{d} x_{2} \wedge \mathrm{d} x_{3} .
\end{gathered}
$$

Note that from the above formulas, there is an obvious identification of $\Lambda^{0}(\mathbb{V} ; \mathbb{K})$ and $\Lambda^{3}(\mathbb{V} ; \mathbb{K})$ with the space of 3 -dimensional vectors and of $\Lambda^{1}(\mathbb{V} ; \mathbb{K})$ and $\Lambda^{2}(\mathbb{V} ; \mathbb{K})$ with $3 \times 3$ matrices (again with $W_{i j}=w_{i j}$ in both cases).

In the mixed formulation of elasticity, we shall need a special operator $S=$ $S_{k}: \Lambda^{k}(\mathbb{V}, \mathbb{V}) \rightarrow \Lambda^{k+1}(\mathbb{V}, \mathbb{K})$ defined as follows: First define $K_{k}: \Lambda^{k}(\Omega ; \mathbb{V}) \rightarrow$ $\Lambda^{k}(\Omega ; \mathbb{K})$ by

$$
K_{k} \omega=X \omega^{T}-\omega X^{T},
$$

where $X=\left(x_{1}, \cdots, x_{n}\right)^{T}$. Then define

$$
S_{k}=\mathrm{d}_{k} K_{k}-K_{k+1} \mathrm{~d}_{k}: \Lambda^{k}(\Omega ; \mathbb{V}) \rightarrow \Lambda^{k+1}(\Omega ; \mathbb{K}) .
$$

Using the definition of the exterior derivative, the definition of $K$, and the Leibniz rule, one can show that for any vector $\left(v_{1}, \ldots, v_{k+1}\right)$, 


$$
\begin{aligned}
& \left(S_{k} \omega\right)_{x}\left(v_{1}, \ldots, v_{k+1}\right) \\
& =\sum_{j=1}^{k+1}(-1)^{j+1}\left[v_{j} \omega^{T}\left(v_{1}, \cdots, \hat{v}_{j}, \cdots, v_{k+1}\right)-\omega\left(v_{1}, \cdots, \hat{v}_{j}, \cdots, v_{k+1}\right) v_{j}^{T}\right]
\end{aligned}
$$

where the notation $\hat{v}_{j}$ means that this argument is omitted. Thus, $S_{k}$ is a purely algebraic operator.

More specifically, we shall need this operator when $k=n-2$ and $k=n-1$. We examine these cases below for $n=2$ and $n=3$. When $n=2$, we get for $\omega=\left(w_{1}, w_{2}\right)^{T}, K_{0} \omega=\left(w_{1} x_{2}-w_{2} x_{1}\right) \chi$, and after a simple computation,

$$
S_{0} \omega=\left(\mathrm{d}_{0} K_{0}-K_{1} \mathrm{~d}_{0}\right) \omega=-w_{2} \chi \mathrm{d} x_{1}+w_{1} \boldsymbol{\chi} \mathrm{d} x_{2} .
$$

Note that $S_{0}$ is invertible with

$$
S_{0}^{-1}\left[\mu_{1} \chi \mathrm{d} x_{1}+\mu_{2} \chi \mathrm{d} x_{2}\right]=\left(\mu_{2},-\mu_{1}\right)^{T} .
$$

If $\omega \in \Lambda^{1}(\mathbb{V} ; \mathbb{V})$ is given by:

$$
\omega=w_{1} \mathrm{~d} x_{1}+w_{2} \mathrm{~d} x_{2}, \quad w_{1}=\left(w_{11}, w_{21}\right)^{T}, \quad w_{2}=\left(w_{12}, w_{22}\right)^{T},
$$

then $S_{1} \omega=-\left(w_{11}+w_{22}\right) \chi \mathrm{d} x_{1} \wedge \mathrm{d} x_{2}$. If we identity $\omega$ with a matrix $W$ by

$$
\left(\begin{array}{ll}
W_{11} & W_{12} \\
W_{21} & W_{22}
\end{array}\right)=\left(\begin{array}{ll}
-w_{12} & w_{11} \\
-w_{22} & w_{21}
\end{array}\right)
$$

then we can identify $S_{1} \omega$ with the matrix

$$
\left(\begin{array}{cc}
0 & W_{12}-W_{21} \\
W_{21}-W_{12} & 0
\end{array}\right)=W-W^{T} \equiv 2 \operatorname{skw} W .
$$

When $n=3$, we get for $\omega=w_{1} \mathrm{~d} x_{1}+w_{2} \mathrm{~d} x_{2}+w_{3} \mathrm{~d} x_{3}$, with $w_{j}=$ $\left(w_{1 j}, w_{2 j}, w_{3 j}\right)^{T}$,

$$
\begin{aligned}
S_{1} \omega=\operatorname{Skw}\left(-w_{33}-w_{22}, w_{12}, w_{13}\right) \mathrm{d} x_{2} \wedge \mathrm{d} x_{3} \\
-\operatorname{Skw}\left(w_{21},-w_{11}-w_{33}, w_{23}\right) \mathrm{d} x_{1} \wedge \mathrm{d} x_{3} \\
+\operatorname{Skw}\left(w_{31}, w_{32},-w_{11}-w_{22}\right) \mathrm{d} x_{1} \wedge \mathrm{d} x_{2} .
\end{aligned}
$$

If we identify $\omega \in \Lambda^{1}(\mathbb{V} ; \mathbb{V})$ with a matrix $W$ by $W_{i j}=w_{i j}$, and identify $S_{1} \omega \in \Lambda^{2}(\mathbb{V} ; \mathbb{K})$ with the matrix $U$ given by

$$
U=\left(\begin{array}{ccc}
-w_{33}-w_{22} & w_{21} & w_{31} \\
w_{12} & -w_{11}-w_{33} & w_{32} \\
w_{13} & w_{23} & -w_{11}-w_{22}
\end{array}\right),
$$

then, $W$ and $U$ are related by the equations

$$
U=\Xi W \equiv W^{T}-\operatorname{tr}(W) I, \quad W=\Xi^{-1} U \equiv U^{T}-\frac{1}{2} \operatorname{tr}(U) I .
$$


Hence, $S_{1}$ is invertible.

$$
\begin{aligned}
& \text { If } \omega=w_{1} \mathrm{~d} x_{2} \wedge \mathrm{d} x_{3}-w_{2} \mathrm{~d} x_{1} \wedge \mathrm{d} x_{3}+w_{3} \mathrm{~d} x_{1} \wedge \mathrm{d} x_{2} \text {, then } \\
& \qquad S_{2} \omega=\left(\begin{array}{ccc}
0 & w_{21}-w_{12} & w_{31}-w_{13} \\
w_{12}-w_{21} & 0 & w_{32}-w_{23} \\
w_{13}-w_{31} & w_{23}-w_{32} & 0
\end{array}\right) \mathrm{d} x_{1} \wedge \mathrm{d} x_{2} \wedge \mathrm{d} x_{3} .
\end{aligned}
$$

If we identify $\omega$ with the matrix $W$ given by $W_{i j}=w_{i j}$, then by the above, $S_{2} \omega$ may be identified with the matrix -2 skw $W$.

We easily obtain from the fact that $\mathrm{d}_{k+1} \mathrm{~d}_{k}=0$ and the definition $S_{k}=$ $\mathrm{d}_{k} K_{k}-K_{k+1} \mathrm{~d}_{k}$ that

$$
\mathrm{d}_{k+1} S_{k}+S_{k+1} \mathrm{~d}_{k}=0 .
$$

This identify, for $k=n-2$, i.e., $\mathrm{d}_{n-1} S_{n-2}+S_{n-1} \mathrm{~d}_{n-2}=0$ is the key identity in establishing stability of continuous and discrete variational formulations of elasticity with weak symmetry.

Note that this formula is much more complicated and also different in different dimensions when stated in terms of proxy fields (which are reasons why we have introduced differential forms). When $n=2$ and $k=0$, if we identify $\omega=\left(w_{1}, w_{2}\right)^{T} \in \Lambda^{0}(\Omega ; \mathbb{V})$ with the vector $W$, then the formula $\left(\mathrm{d}_{1} S_{0}+S_{1} \mathrm{~d}_{0}\right) \omega=0$ becomes

$$
(\operatorname{div} W) \chi+2 \operatorname{skw} \operatorname{curl} W=0 .
$$

When $n=3$ and $k=1$, if we identify $\omega \in \Lambda^{1}(\Omega ; \mathbb{V})$ with the matrix $W$, then the formula $\left(\mathrm{d}_{2} S_{1}+S_{2} \mathrm{~d}_{1}\right) \omega=0$ becomes

$$
\operatorname{Skw} \operatorname{div}(\Xi W)-2 \operatorname{skw} \operatorname{curl} W=0 .
$$

\section{Mixed formulation of the equations of elasticity with weak symmetry}

In order to write 3 in the language of exterior calculus, we will use the spaces of vector-valued differential forms presented in the previous section. We assume that $\Omega$ is a contractible domain in $\mathbb{R}^{n}, \mathbb{V}=\mathbb{R}^{n}$, and $\mathbb{K}$ is again the space of skew-symmetric matrices. We showed in the last section that the operator $S=$ $S_{n-1}: \Lambda^{n-1}(\Omega ; \mathbb{V}) \rightarrow \Lambda^{n}(\Omega ; \mathbb{K})$ corresponds (up to a factor of \pm 2 ) to taking the skew-symmetric part of its argument. Setting $\mathrm{d}_{n-1}=\mathrm{d}$, the elasticity problem 3 becomes: Find $(\sigma, u, p) \in H \Lambda^{n-1}(\Omega ; \mathbb{V}) \times L^{2} \Lambda^{n}(\Omega ; \mathbb{V}) \times L^{2} \Lambda^{n}(\Omega ; \mathbb{K})$ such that

$$
\begin{gathered}
\langle A \sigma, \tau\rangle+\langle\mathrm{d} \tau, u\rangle-\langle S \tau, p\rangle=0, \quad \tau \in H \Lambda^{n-1}(\Omega ; \mathbb{V}), \\
\langle\mathrm{d} \sigma, v\rangle=\langle f, v\rangle, \quad v \in L^{2} \Lambda^{n}(\Omega ; \mathbb{V}), \quad\langle S \sigma, q\rangle=0, \quad q \in L^{2} \Lambda^{n}(\Omega ; \mathbb{K}) .
\end{gathered}
$$

This problem is well-posed in the sense that, for each $f \in L^{2} \Lambda^{n}(\Omega ; \mathbb{V})$, there exists a unique solution $(\sigma, u, p) \in H \Lambda^{n-1}(\Omega ; \mathbb{V}) \times L^{2} \Lambda^{n}(\Omega ; \mathbb{V}) \times L^{2} \Lambda^{n}(\Omega ; \mathbb{K})$, and the solution operator is a bounded operator 


$$
L^{2} \Lambda^{n}(\Omega ; \mathbb{V}) \rightarrow H \Lambda^{n-1}(\Omega ; \mathbb{V}) \times L^{2} \Lambda^{n}(\Omega ; \mathbb{V}) \times L^{2} \Lambda^{n}(\Omega ; \mathbb{K}) .
$$

This will follow from the general theory of such saddle point problems [14] once we establish two conditions:

(W1) $\|\tau\|_{H \Lambda}^{2} \leq c_{1}\langle A \tau, \tau\rangle$ whenever $\tau \in H \Lambda^{n-1}(\Omega ; \mathbb{V})$ satisfies $\langle\mathrm{d} \tau, v\rangle=0$ $\forall v \in L^{2} \Lambda^{n}(\Omega ; \mathbb{V})$ and $\langle S \tau, q\rangle=0 \forall q \in L^{2} \Lambda^{n}(\Omega ; \mathbb{K})$,

(W2) for all nonzero $(v, q) \in L^{2} \Lambda^{n}(\Omega ; \mathbb{V}) \times L^{2} \Lambda^{n}(\Omega ; \mathbb{K})$, there exists nonzero $\tau \in H \Lambda^{n-1}(\Omega ; \mathbb{V})$ with $\langle\mathrm{d} \tau, v\rangle-\langle S \tau, q\rangle \geq c_{2}\|\tau\|_{H \Lambda}(\|v\|+\|q\|)$,

for some positive constants $c_{1}$ and $c_{2}$. The first condition is obvious (and does not even utilize the orthogonality of $S \tau$ ). However, the second condition is more subtle. We will verify it in Theorem 7.2 in a subsequent section.

We next consider a finite element discretizations of 11 . For this, we choose families of finite-dimensional subspaces

$$
\Lambda_{h}^{n-1}(\mathbb{V}) \subset H \Lambda^{n-1}(\Omega ; \mathbb{V}), \quad \Lambda_{h}^{n}(\mathbb{V}) \subset L^{2} \Lambda^{n}(\Omega ; \mathbb{V}), \quad \Lambda_{h}^{n}(\mathbb{K}) \subset L^{2} \Lambda^{n}(\Omega ; \mathbb{K}),
$$

indexed by $h$, and seek the discrete solution $\left(\sigma_{h}, u_{h}, p_{h}\right) \in \Lambda_{h}^{n-1}(\mathbb{V}) \times \Lambda_{h}^{n}(\mathbb{V}) \times$ $\Lambda_{h}^{n}(\mathbb{K})$ such that

$$
\begin{gathered}
\left\langle A \sigma_{h}, \tau\right\rangle+\left\langle\mathrm{d} \tau, u_{h}\right\rangle-\left\langle S \tau, p_{h}\right\rangle=0, \quad \tau \in \Lambda_{h}^{n-1}(\mathbb{V}), \\
\left\langle\mathrm{d} \sigma_{h}, v\right\rangle=\langle f, v\rangle \quad v \in \Lambda_{h}^{n}(\mathbb{V}), \quad\left\langle S \sigma_{h}, q\right\rangle=0, \quad q \in \Lambda_{h}^{n}(\mathbb{K}) .
\end{gathered}
$$

In analogy with the well-posedness of the problem 11, the stability of the saddle point system 12 will be ensured by the Brezzi stability conditions:

(S1) $\|\tau\|_{H \Lambda}^{2} \leq c_{1}(A \tau, \tau)$ whenever $\tau \in \Lambda_{h}^{n-1}(\mathbb{V})$ satisfies $\langle\mathrm{d} \tau, v\rangle=0$ $\forall v \in \Lambda_{h}^{n}(\mathbb{V})$ and $\langle S \tau, q\rangle=0 \forall q \in \Lambda_{h}^{n}(\mathbb{K})$,

(S2) for all nonzero $(v, q) \in \Lambda_{h}^{n}(\mathbb{V}) \times \Lambda_{h}^{n}(\mathbb{K})$, there exists nonzero $\tau \in \Lambda_{h}^{n-1}(\mathbb{V})$ with $\langle\mathrm{d} \tau, v\rangle-\langle S \tau, q\rangle \geq c_{2}\|\tau\|_{H \Lambda}(\|v\|+\|q\|)$,

where now the constants $c_{1}$ and $c_{2}$ must be independent of $h$. The difficulty is, of course, to design finite element spaces satisfying these conditions.

We have seen previously that there is a close relation between the construction of stable mixed finite element methods for the approximation of the equations of linear elasticity and discretization of the associated elasticity complex 6. This relationship extends an analogous relationship between the construction of stable mixed finite element methods for Poisson's equation and discretization of the de Rham complex. It turns out that there is also a close, but non-obvious, connection between the elasticity complex and the de Rham complex. This connection is described in [19] and is related to a general construction given in [13], called the BGG resolution (see also [16]).

The elasticity complex 6 is related to the formulation of the equations of elasticity with strong symmetry. It is also possible to derive an elasticity complex that is related to the equations of elasticity with weak symmetry, again starting from the de Rham complex. In [8] (two dimensions) and [6] 
(three dimensions), such an elasticity complex is derived and a discrete version of the BGG construction also developed. This was then used to derive stable mixed finite element methods for elasticity in a systematic manner based on the finite element versions of the de Rham sequence described earlier. The resulting elements in both two and three space dimensions are simpler than any derived previously. For example, the simple choice of $\mathcal{P}_{1} \Lambda^{n-1}\left(\mathcal{T}_{h} ; \mathbb{V}\right)$ for stress, $\mathcal{P}_{0} \Lambda^{n}\left(\mathcal{T}_{h} ; \mathbb{V}\right)$ for displacement, and $\mathcal{P}_{0} \Lambda^{n}\left(\mathcal{T}_{h} ; \mathbb{K}\right)$ for the multiplier results in a stable discretization of the problem 12. In Figure 4, this element is depicted in two dimensions. For stress, the degrees of freedom are the first two moments of its trace on the edges, and for the displacement and multiplier, their integrals on the triangle (two components for displacement, one for the multiplier). Moreover, this element is the lowest order of a family of stable elements in $n$ dimensions utilizing $\mathcal{P}_{r} \Lambda^{n-1}\left(\mathcal{T}_{h} ; \mathbb{V}\right)$ for stress, $\mathcal{P}_{r-1} \Lambda^{n}\left(\mathcal{T}_{h} ; \mathbb{V}\right)$ for displacement, and $\mathcal{P}_{r-1} \Lambda^{n}\left(\mathcal{T}_{h} ; \mathbb{K}\right)$ for the multiplier. In fact, the lowest order element may be simplified further, so that only a subset of linear vectors is needed to approximate the stress. More details of this simplified element are presented in Section 11.
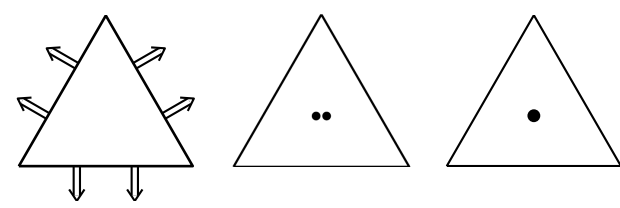

Fig. 4. Approximation of stress, displacement, and multiplier for the simplest element in two dimensions.

In the next section, we follow the approach in [9] and outline how an elasticity complex with weakly imposed symmetry can be derived from the de Rham complex. Since this derivation produces a sequence in the notation of differential forms, we then translate our results to the more classical notation for elasticity in two and three dimensions. In Section 7, we give a proof of the well-posedness of the mixed formulation of elasticity with weak symmetry for the continuous problem, as a guide for establishing a similar result for the discrete problem. Based on this proof, we develop in Section 8 the conditions that we will need for stable approximation schemes. These results are then used to establish the main stability result for weakly symmetric mixed finite element approximations of the equations of elasticity in Section 9 and some more refined estimates in Section 10. The results presented in this paper are for the case of displacement boundary conditions. An extension to the equations of elasticity with traction boundary conditions can be found in [9]. 


\section{From the de Rham complex to an elasticity complex with weak symmetry}

In this section, we discuss the connection of the elasticity complex in $n$ dimensions with the de Rham complex. Details of the derivation can be found in $[6]$ and [9] and follow the ideas in a a derivation of elasticity from the de Rham sequence in the case of strongly imposed symmetry given in [19] in three dimensions.

We start with the two vector-valued de Rham sequences, one with values in $\mathbb{V}$ and one with values in $\mathbb{K}$, i.e.,

$$
\begin{gathered}
\Lambda^{n-2}(\Omega ; \mathbb{K}) \stackrel{\mathrm{d}_{n-2}}{\longrightarrow} \Lambda^{n-1}(\Omega ; \mathbb{K}) \stackrel{\mathrm{d}_{n-1}}{\longrightarrow} \Lambda^{n}(\mathbb{K}) \rightarrow 0, \\
\Lambda^{n-3}(\Omega ; \mathbb{V}) \stackrel{\mathrm{d}_{n-3}}{\longrightarrow} \Lambda^{n-2}(\Omega ; \mathbb{V}) \stackrel{\mathrm{d}_{n-2}}{\longrightarrow} \Lambda^{n-1}(\Omega ; \mathbb{V}) \stackrel{\mathrm{d}_{n-1}}{\longrightarrow} \Lambda^{n}(\mathbb{V}) \rightarrow 0,
\end{gathered}
$$

Using the fact that these sequences are exact, one is able to show that the sequence

$$
\begin{aligned}
\Lambda^{n-3}(\mathbb{W}) \stackrel{\left(\mathrm{d}_{n-3},-S_{n-3}\right)}{\longrightarrow} \Lambda^{n-2}(\Omega ; \mathbb{K}) \stackrel{\mathrm{d}_{n-2} \circ S_{n-2}^{-1} \circ \mathrm{d}_{n-2}}{\longrightarrow} \Lambda^{n-1}(\Omega ; \mathbb{V}) \\
\stackrel{\left(-S_{n-1}, \mathrm{~d}_{n-1}\right)^{T}}{\longrightarrow} \Lambda^{n}(\mathbb{W}) \rightarrow 0
\end{aligned}
$$

is exact, where $\mathbb{W}=\mathbb{K} \times \mathbb{V}$. We refer to the sequence 13 as the elasticity sequence with weak symmetry. Crucial to this construction is the fact that the operator $S_{n-2}: H^{1} \Lambda^{n-2}(\Omega ; \mathbb{V}) \rightarrow H^{1} \Lambda^{n-1}(\Omega ; \mathbb{K})$ is an isomorphism.

We next interpret this sequence in the language of differential operators in two and three dimensions. When $n=2$, we have the sequence

$$
\Lambda^{0}(\Omega ; \mathbb{K}) \stackrel{\mathrm{d}_{0} \circ S_{0}^{-1} \circ \mathrm{d}_{0}}{\longrightarrow} \Lambda^{1}(\Omega ; \mathbb{V}) \stackrel{\left(-S_{1}, \mathrm{~d}_{1}\right)^{T}}{\longrightarrow} \Lambda^{2}(\mathbb{W}) \rightarrow 0 .
$$

Hence, if we begin with an element $w \chi \in \Lambda^{0}(\Omega ; \mathbb{K})$ that we identify with the scalar function $w$, then

$$
\begin{gathered}
\mathrm{d}_{0}(w \boldsymbol{\chi})=\frac{\partial w}{\partial x_{1}} \boldsymbol{\chi} \mathrm{d} x_{1}+\frac{\partial w}{\partial x_{2}} \boldsymbol{\chi} \mathrm{d} x_{2}, \quad S_{0}^{-1}\left[\mathrm{~d}_{0}(w \boldsymbol{\chi})\right]=\left(\frac{\partial w}{\partial x_{2}},-\frac{\partial w}{\partial x_{1}}\right)^{T}, \\
\mathrm{~d}_{0} S_{0}^{-1}\left[\mathrm{~d}_{0}(w \boldsymbol{\chi})\right]=\left(\begin{array}{c}
\partial^{2} w / \partial x_{1} \partial x_{2} \\
-\partial^{2} w / \partial x_{1}^{2}
\end{array}\right) \mathrm{d} x_{1}+\left(\begin{array}{c}
\partial^{2} w / \partial x_{2}^{2} \\
-\partial^{2} w / \partial x_{1} \partial x_{2}
\end{array}\right) \mathrm{d} x_{2} .
\end{gathered}
$$

We then identity this vector-valued 1-form with the matrix

$$
\left(\begin{array}{cc}
-\partial^{2} w / \partial x_{2}^{2} & \partial^{2} w / \partial x_{1} \partial x_{2} \\
\partial^{2} w / \partial x_{1} \partial x_{2} & -\partial^{2} w / \partial x_{1}^{2}
\end{array}\right) \equiv-J w
$$

To translate the second part of the sequence, we begin with an element $\omega=$ $\left(\begin{array}{l}w_{11} \\ w_{21}\end{array}\right) \mathrm{d} x_{1}+\left(\begin{array}{l}w_{12} \\ w_{22}\end{array}\right) \mathrm{d} x_{2} \in \Lambda^{1}(\mathbb{V} ; \mathbb{V})$ that we identify (as in 10) with the matrix 


$$
W=\left(\begin{array}{ll}
W_{11} & W_{12} \\
W_{21} & W_{22}
\end{array}\right)=\left(\begin{array}{ll}
-w_{12} & w_{11} \\
-w_{22} & w_{21}
\end{array}\right)
$$

We have seen previously that $-S_{1} \omega$ corresponds to $-2 \mathrm{skw} W$. Now

$$
\mathrm{d}_{1} \omega=\left(\begin{array}{l}
\partial w_{12} / \partial x_{1}-\partial w_{11} / \partial x_{2} \\
\partial w_{22} / \partial x_{1}-\partial w_{21} / \partial x_{2}
\end{array}\right) \mathrm{d} x_{1} \wedge \mathrm{d} x_{2}=-\operatorname{div} W \mathrm{~d} x_{1} \wedge \mathrm{d} x_{2} .
$$

Hence, modulo some constants, we obtain the elasticity sequence

$$
C^{\infty}(\Omega) \stackrel{J}{\rightarrow} C^{\infty}(\Omega ; \mathbb{M}) \stackrel{(\text { skw, div })^{T}}{\longrightarrow} C^{\infty}(\Omega, \mathbb{K} \times \mathbb{V}) \rightarrow 0
$$

When $n=3$, we have the sequence

$$
\Lambda^{0}(\mathbb{W}) \stackrel{\left(\mathrm{d}_{0},-S_{0}\right)}{\longrightarrow} \Lambda^{1}(\Omega ; \mathbb{K}) \stackrel{\mathrm{d}_{1} \circ S_{1}^{-1} \circ \mathrm{od}_{1}}{\longrightarrow} \Lambda^{2}(\Omega ; \mathbb{V}) \stackrel{\left(-S_{2}, \mathrm{~d}_{2}\right)^{T}}{\longrightarrow} \Lambda^{3}(\mathbb{W}) \rightarrow 0
$$

Hence, if we begin with a pair $(\operatorname{Skw} w, \mu) \in \Lambda^{0}(\mathbb{W})=\Lambda^{0}(\Omega, \mathbb{K}) \times \Lambda^{0}(\Omega, \mathbb{V})$ that we identify with the pair $(w, \operatorname{Skw} \mu) \in C^{\infty}(\Omega, \mathbb{V}) \times C^{\infty}(\Omega, \mathbb{K})$, then $\mathrm{d}_{0}$ corresponds to the row-wise gradient and $S_{0}$ to the inclusion of $C^{\infty}(\Omega, \mathbb{K}) \rightarrow$ $C^{\infty}(\Omega, \mathbb{M})$. We have discussed previously natural identifications of $\Lambda^{1}(\Omega ; \mathbb{K})$ and $\Lambda^{2}(\Omega ; \mathbb{V})$ with $C^{\infty}(\Omega ; \mathbb{M})$. With these identifications, $\mathrm{d}_{1}$ corresponds to the row-wise curl and $S_{1}$ to the operator $\Xi$. Finally, we have also seen how $-S_{2}$ corresponds to the operator $2 \mathrm{skw}$. Since $\mathrm{d}_{2}$ corresponds to the row-wise divergence, we obtain (modulo some unimportant constants), the elasticity sequence with weak symmetry

$C^{\infty}(\mathbb{V} \times \mathbb{K}) \stackrel{(\text { grad }, I)}{\longrightarrow} C^{\infty}(\mathbb{M}) \stackrel{\text { curl } \Xi^{-1} \text { curl }}{\longrightarrow} C^{\infty}(\mathbb{M}) \stackrel{(\text { skw,div })^{T}}{\longrightarrow} C^{\infty}(\mathbb{K} \times \mathbb{V}) \rightarrow 0$

More details, and the extension of these ideas to more general domains, can be found in [9].

\section{Well-posedness of the weak symmetry formulation of elasticity}

As discussed in Section 5, to establish well-posedness of the elasticity problem with weakly imposed symmetry 11, it suffices to verify condition (W2) of that section. This may be deduced from the following theorem, which says that the map

$$
H \Lambda^{n-1}(\Omega ; \mathbb{V}) \stackrel{\left(-S_{n-1}, \mathrm{~d}_{n-1}\right)^{T}}{\longrightarrow} H \Lambda^{n}(\Omega ; \mathbb{K}) \times H \Lambda^{n}(\Omega ; \mathbb{V})
$$

is surjective. We present the proof in detail, since it will give us guidance as we construct stable discretizations. The proof will make use of the following well-known result from partial differential equations. 
Lemma 7.1 Let $\Omega$ be a bounded domain in $\mathbb{R}^{n}$ with a Lipschitz boundary. Then, for all $\mu \in L^{2} \Lambda^{n}(\Omega)$, there exists $\eta \in H^{1} \Lambda^{n-1}(\Omega)$ satisfying $\mathrm{d}_{n-1} \eta=\mu$. If, in addition, $\int_{\Omega} \mu=0$, then we can choose $\eta \in \stackrel{\circ}{H}^{1} \Lambda^{n-1}(\Omega)$.

Theorem 7.2 Given $(\omega, \mu) \in L^{2} \Lambda^{n}(\Omega ; \mathbb{K}) \times L^{2} \Lambda^{n}(\Omega ; \mathbb{V})$, there exists $\sigma \in$ $H \Lambda^{n-1}(\Omega ; \mathbb{V})$ such that $\mathrm{d}_{n-1} \sigma=\mu,-S_{n-1} \sigma=\omega$. Moreover, we may choose $\sigma$ so that

$$
\|\sigma\|_{H \Lambda} \leq c(\|\omega\|+\|\mu\|)
$$

for a fixed constant $c$.

Proof. The second sentence follows from the first by Banach's theorem, (i.e., if a continuous linear operator between two Banach spaces has an inverse, then this inverse operator is continuous), so we need only prove the first.

(1) By Lemma 7.1, we can find $\eta \in H^{1} \Lambda^{n-1}(\Omega ; \mathbb{V})$ with $\mathrm{d}_{n-1} \eta=\mu$.

(2) Since $\omega+S_{n-1} \eta \in H \Lambda^{n}(\Omega ; K)$, we can apply Lemma 7.1 a second time to find $\tau \in H^{1} \Lambda^{n-1}(\Omega ; \mathbb{K})$ with $\mathrm{d}_{n-1} \tau=\omega+S_{n-1} \eta$.

(3) Since $S_{n-2}$ is an isomorphism from $H^{1} \Lambda^{n-2}(\Omega ; \mathbb{V})$ onto $H^{1} \Lambda^{n-1}(\Omega ; \mathbb{K})$, we have $\varrho \in H^{1} \Lambda^{n-2}(\Omega ; \mathbb{V})$ with $S_{n-2} \varrho=\tau$.

(4) Define $\sigma=\mathrm{d}_{n-2} \varrho+\eta \in H \Lambda^{n-1}(\Omega ; \mathbb{V})$.

(5) From steps (1) and (4), it is immediate that $\mathrm{d}_{n-1} \sigma=\mu$.

(6) From (4), $-S_{n-1} \sigma=-S_{n-1} \mathrm{~d}_{n-2} \varrho-S_{n-1} \eta$. But, since $\mathrm{d}_{n-1} S_{n-2}=$ $-S_{n-1} \mathrm{~d}_{n-2}$,

$$
-S_{n-1} \mathrm{~d}_{n-2} \varrho=\mathrm{d}_{n-1} S_{n-2} \varrho=\mathrm{d}_{n-1} \tau=\omega+S_{n-1} \eta,
$$

so $-S_{n-1} \sigma=\omega$.

We note a few points from the proof.

(i) Although the elasticity problem 11 only involves the three spaces $H \Lambda^{n-1}(\Omega ; \mathbb{V}), L^{2} \Lambda^{n}(\Omega ; \mathbb{V})$, and $L^{2} \Lambda^{n}(\Omega ; \mathbb{K})$, the proof brings in two additional spaces from the BGG construction: $H \Lambda^{n-2}(\Omega ; \mathbb{V})$ and $H \Lambda^{n-1}(\Omega ; \mathbb{K})$.

(ii) Although $S_{n-1}$ is the only $S$ operator arising in the formulation, $S_{n-2}$ plays a role in the proof.

(iii) We do not fully use the fact that $S_{n-2}$ is an isomorphism from $\Lambda^{n-2}(\mathbb{V} ; \mathbb{V})$ to $\Lambda^{n-1}(\mathbb{V} ; \mathbb{K})$, only the fact that it is a surjection. This will prove important in the next section, when we derive conditions for stable approximation schemes for elasticity.

(iv) Other slightly weaker conditions can be used in some places in the proof (a fact we also exploit in discrete versions for some choices of finite element spaces).

\section{Conditions for stable approximation schemes}

To obtain stable approximation schemes, we now mimic the key structural elements present for the continuous problem. In particular, we see that to 
establish stability of the continuous problem, we do not use the complete exact sequences, but only the last two spaces in the top sequence and the last three spaces in the bottom sequence, connected by the operators $S_{n-2}$ and $S_{n-1}$.

$$
\begin{gathered}
\Lambda^{n-1}(\mathbb{K}) \stackrel{\mathrm{d}_{n-1}}{\longrightarrow} \Lambda^{n}(\mathbb{K}) \rightarrow 0 \\
\nearrow S_{n-2} \nearrow S_{n-1} \\
\Lambda^{n-2}(\mathbb{V}) \stackrel{\mathrm{d}_{n-2}}{\longrightarrow} \Lambda^{n-1}(\mathbb{V}) \stackrel{\mathrm{d}_{n-1}}{\longrightarrow} \Lambda^{n}(\mathbb{V}) \rightarrow 0 .
\end{gathered}
$$

Thus, we look for five finite dimensional spaces that are connected by a similar structure, i.e., in addition to the spaces $\Lambda_{h}^{n}(\mathbb{K}) \subset H \Lambda^{n}(\mathbb{K}), \Lambda_{h}^{n-1}(\mathbb{V}) \subset$ $H \Lambda^{n-1}(\mathbb{V})$, and $\Lambda_{h}^{n}(\mathbb{V}) \subset H \Lambda^{n}(\mathbb{V})$ used in the finite element method, we also seek spaces $\Lambda_{h}^{n-1}(\mathbb{K}) \subset H \Lambda^{n-1}(\mathbb{K})$ and $\Lambda_{h}^{n-2}(\mathbb{V}) \subset H \Lambda^{n-2}(\mathbb{V})$.

To mimic the structure of the continuous problem, but taking into account the comments made following Theorem 7.2, we require that the finite element spaces are also connected by exact sequences, but where we introduce some additional flexibility by inserting the $L^{2}$ projection operator $\Pi_{h}^{n}$ and using approximations of the operators $S_{n-2}$ and $S_{n-1}$.

$$
\begin{gathered}
\Lambda_{h}^{n-1}(\mathbb{K}) \stackrel{\Pi_{h}^{n} \mathrm{~d}_{n-1}}{\longrightarrow} \Lambda_{h}^{n}(\mathbb{K}) \rightarrow 0 \\
\nearrow S_{n-2, h} S_{n-1, h} \\
\Lambda_{h}^{n-2}(\mathbb{V}) \stackrel{\mathrm{d}_{n-2}}{\longrightarrow} \Lambda_{h}^{n-1}(\mathbb{V}) \stackrel{\mathrm{d}_{n-1}}{\longrightarrow} \Lambda_{h}^{n}(\mathbb{V}) \rightarrow 0 .
\end{gathered}
$$

In anticipation of proving a stability result for the mixed finite element method for elasticity that mimics that proof used in the continuous case, we need to define interpolants into each of these finite element spaces that have appropriate properties. The reason for the choice of the specific properties will become apparent in the stability proof.

We first define $\Pi_{h}^{n}$ and $\tilde{\Pi}_{h}^{n}$ to be the $L^{2}$ projection operators into the spaces $\Lambda_{h}^{n}(\mathbb{K})$ and $\Lambda_{h}^{n}(\mathbb{V})$, respectively. We then define $\Pi_{h}^{n-1}$ and $\tilde{\Pi}_{h}^{n-1}$ to be interpolation operators mapping $H^{1} \Lambda^{n-1}(\mathbb{K})$ to $\Lambda_{h}^{n-1}(\mathbb{K})$ and $H^{1} \Lambda^{n-1}(\mathbb{V})$ to $\Lambda_{h}^{n-1}(\mathbb{V})$, respectively, and satisfying

$$
\begin{gathered}
\Pi_{h}^{n} \mathrm{~d}_{n-1} \Pi_{h}^{n-1} \tau=\Pi_{h}^{n} \mathrm{~d}_{n-1} \tau, \tau \in\left(\stackrel{\circ}{H}^{1}+P^{1}\right) \Lambda^{n-1}(\mathbb{K}), \\
\mathrm{d}_{n-1} \tilde{\Pi}_{h}^{n-1} \tau=\tilde{\Pi}_{h}^{n} \mathrm{~d}_{n-1} \tau, \tau \in H^{1} \Lambda^{n-1}(\mathbb{V}) . \\
\left\|\Pi_{h}^{n-1} \tau\right\| \leq C\|\tau\|_{1}, \tau \in\left(\stackrel{H}{ }^{1}+P^{1}\right) \Lambda^{n-1}(\mathbb{K}), \\
\left\|\tilde{\Pi}_{h}^{n-1} \tau\right\| \leq C\|\tau\|_{1}, \tau \in H^{1} \Lambda^{n-1}(\mathbb{V}) .
\end{gathered}
$$

Next, we define $\tilde{\Pi}_{h}^{n-2}$ mapping $H^{1} \Lambda^{n-2}(\mathbb{V})$ to $\Lambda_{h}^{n-2}(\mathbb{V})$ satisfying

$$
\left\|\mathrm{d}_{n-2} \tilde{\Pi}_{h}^{n-2} \varrho\right\| \leq c\|\varrho\|_{1}, \quad \varrho \in H^{1} \Lambda^{n-2} .
$$


(In 18, the exterior derivative $\mathrm{d}_{n-2}$ corresponds to the differential operator curl.) As we shall see in the examples, in some cases these will be the canonical interpolation operators we usually associate with standard finite element spaces, while in other cases, we will need to make some modifications so that the interpolation operators are defined on spaces of functions will less smoothness than we usually assume.

The key to the derivation of the formulation of elasticity with weak symmetry at the continuous level was the introduction of the operators $S=S_{k}: \Lambda^{k}(\mathbb{V}) \rightarrow \Lambda^{k+1}(\mathbb{K})$. In the reduced sequence 14 , only the operators $S_{n-2}$ and $S_{n-1}$ will enter the analysis. One of the key properties of these operators was that

$$
\mathrm{d}_{n-1} S_{n-2}=-S_{n-1} \mathrm{~d}_{n-2} .
$$

For the discrete version of this analysis, we will need to modify the definitions of $S_{n-2}$ and $S_{n-1}$ in a simple way. As a discrete analogue of the operator $S_{n-1}$, we define $S_{n-1, h}: \Lambda_{h}^{n-1}(\mathbb{V}) \rightarrow \Lambda_{h}^{3}(\mathbb{K})$ by $S_{n-1, h}=\Pi_{h}^{n} S_{n-1}$. As a discrete analogue of the operator $S_{n-2}$, we define $S_{n-2, h}: \Lambda_{h}^{n-2}(\mathbb{V}) \rightarrow \Lambda_{h}^{2}(\mathbb{K})$ by $S_{n-2, h}=\Pi_{h}^{n-1} S_{n-2}$. With these definitions, we establish the following discrete version of 19 ,

$$
\Pi_{h}^{n} \mathrm{~d}_{n-1} S_{n-2, h}=-S_{n-1, h} \mathrm{~d}_{n-2} .
$$

To see this, we observe that using 16 and 20,

$$
\begin{aligned}
\Pi_{h}^{n} \mathrm{~d}_{n-1} S_{n-2, h}=\Pi_{h}^{n} \mathrm{~d}_{n-1} \Pi_{h}^{n-1} S_{n-2} & =\Pi_{h}^{n} \mathrm{~d}_{n-1} S_{n-2} \\
& =-\Pi_{h}^{n} S_{n-1} \mathrm{~d}_{n-2}=-S_{n-1, h} \mathrm{~d}_{n-2} .
\end{aligned}
$$

Another key property of the operator $S_{n-2}$ was that it was invertible as a map from $H^{1} \Lambda^{n-2}(\mathbb{V})$ to $H^{1} \Lambda^{n-1}(\mathbb{K})$. This fact was used in the prove of stability of the weak symmetry formulation at the continuous level, although we observed that surjectivity of this map would be sufficient. We cannot expect invertibility of the map $S_{n-2, h}$ However, a key condition to prove stability of the finite element approximation to the weak symmetry formulation is that $S_{n-2, h} \operatorname{maps} \Lambda_{h}^{n-2}(\mathbb{V})$ onto $\Lambda_{h}^{n-1}(\mathbb{K})$. To ensure this condition, we will assume that $\Lambda_{h}^{n-2}(\mathbb{V})$ and $\Lambda_{h}^{n-1}(\mathbb{K})$ are related by the condition.

$$
S_{n-2, h} \tilde{\Pi}_{h}^{n-2} \tau=\Pi_{h}^{n-1} S_{n-2} \tau, \quad \tau \in H^{1} \Lambda^{n-2}(\mathbb{V}) .
$$

To see that this condition ensures surjectivity, note that given a function $\sigma_{h} \in \Lambda_{h}^{n-1}(\mathbb{K})$, we can find $\sigma \in H^{1} \Lambda^{n-1}(\mathbb{K})$ (e.g., a continuous piecewise polynomial differential form), such that $\sigma_{h}=\Pi_{h}^{n-1} \sigma$. Defining $\tau=S_{n-2}^{-1} \sigma$ and $\tau_{h}=\tilde{\Pi}_{h}^{n-2} \tau \in \Lambda_{h}^{n-2}(\mathbb{V})$, we find that

$$
\sigma_{h}=\Pi_{h}^{n-1} \sigma=\Pi_{h}^{n-1} S_{n-2} \tau=S_{n-2, h} \tilde{\Pi}_{h}^{n-2} \tau=S_{n-2, h} \tau_{h} .
$$

To summarize the results of this section, we will develop stable mixed finite element approximation schemes by finding five finite element spaces. The 
three spaces $\Lambda_{h}^{n}(\mathbb{K}) \subset H \Lambda^{n}(\mathbb{K}), \Lambda_{h}^{n-1}(\mathbb{V}) \subset H \Lambda^{n-1}(\mathbb{V}), \Lambda_{h}^{n}(\mathbb{V}) \subset H \Lambda^{n}(\mathbb{V})$ are used in the method and the spaces $\Lambda_{h}^{n-1}(\mathbb{K}) \subset H \Lambda^{n-1}(\mathbb{K})$ and $\Lambda_{h}^{n-2}(\mathbb{V}) \subset$ $H \Lambda^{n-2}(\mathbb{V})$ are auxiliary spaces crucial to the proof of stability. Associated with each of these spaces is an operator for which we need properties 16 , 17 , and 18. We further assume that the five spaces are connected by the exact sequences given in 15 . Finally, we require 21, which ensures that $S_{n-2, h}$ maps $\Lambda_{h}^{n-2}(\mathbb{V})$ onto $\Lambda_{h}^{n-1}(\mathbb{K})$. Under these conditions, we can then prove the following stability result for the mixed finite element method for elasticity.

\section{Stability of finite element approximation schemes}

Theorem 9.1 Assume that the finite element subspaces $\Lambda_{h}^{k}(\mathbb{K})$ and $\Lambda_{h}^{k}(\mathbb{V})$ are connected by the exact sequences given in 15, that there are operators associated with these subspaces satisfying conditions 16, 17, 18, and that condition 21 is satisfied. Then, given $(\omega, \mu) \in \Lambda_{h}^{n}(\mathbb{K}) \times \Lambda_{h}^{n}(\mathbb{V})$, there exists $\sigma \in \Lambda_{h}^{n-1}(\mathbb{V})$ such that $\mathrm{d}_{n-1} \sigma=\mu,-S_{n-1, h} \sigma \equiv-\Pi_{h}^{n} S_{n-1} \sigma=\omega$, and

$$
\|\sigma\|_{H \Lambda} \leq c(\|\omega\|+\|\mu\|),
$$

where the constant $c$ is independent of $\omega, \mu$ and $h$.

Before proving this theorem, we note that condition 15 immediately implies that the first Brezzi condition (S1) is satisfied and that the second Brezzi condition (S2) easily follows from the conclusion of the theorem.

Proof.

(1) By Lemma 7.1, we can find $\eta \in H^{1} \Lambda^{n-1}(\Omega ; \mathbb{V})$ with $\mathrm{d}_{n-1} \eta=\mu$ and $\|\eta\|_{1} \leq c\|\mu\|$.

(2) Since $\omega+\Pi_{h}^{n} S_{n-1} \tilde{\Pi}_{h}^{n-1} \eta \in H \Lambda^{n}(\Omega ; K)$, we can apply Lemma 7.1 a second time to find $\tau \in H^{1} \Lambda^{n-1}(\Omega ; \mathbb{K})$ with $\mathrm{d}_{n-1} \tau=\omega+\Pi_{h}^{n} S_{n-1} \tilde{\Pi}_{h}^{n-1} \eta$ and $\|\tau\|_{1} \leq c\left(\|\omega\|+\left\|\Pi_{h}^{n} S_{n-1} \tilde{\Pi}_{h}^{n-1} \eta\right\|\right)$.

(3) Since $S_{n-2}$ is an isomorphism from $H^{1} \Lambda^{n-2}(\Omega ; \mathbb{V})$ to $H^{1} \Lambda^{n-1}(\Omega ; \mathbb{K})$, we have $\varrho \in H^{1} \Lambda^{n-2}(\Omega ; \mathbb{V})$ with $S_{n-2} \varrho=\tau$, and $\|\varrho\|_{1} \leq c\|\tau\|_{1}$.

(4) Define $\sigma=\mathrm{d}_{n-2} \tilde{\Pi}_{h}^{n-2} \varrho+\tilde{\Pi}_{h}^{n-1} \eta \in \Lambda_{h}^{n-1}(\mathbb{V})$.

(5) From step (4), 16, step (1), and the fact that $\tilde{\Pi}_{h}^{n}$ is a projection, we have

$$
\mathrm{d}_{n-1} \sigma=\mathrm{d}_{n-1} \tilde{\Pi}_{h}^{n-1} \eta=\tilde{\Pi}_{h}^{n} \mathrm{~d}_{n-1} \eta=\tilde{\Pi}_{h}^{n} \mu=\mu .
$$

(6) Also from step (4),

$$
-S_{n-1, h} \sigma=-S_{n-1, h} \mathrm{~d}_{n-2} \tilde{\Pi}_{h}^{n-2} \varrho-S_{n-1, h} \tilde{\Pi}_{h}^{n-1} \eta .
$$

Applying, in order, 20, 21, step (3), 16, step (2), and the fact that $\Pi_{h}^{n}$ is a projection, we obtain 


$$
\begin{aligned}
& S_{n-1, h} \mathrm{~d}_{n-2} \tilde{\Pi}_{h}^{n-2} \varrho=-\Pi_{h}^{n} \mathrm{~d}_{n-2} S_{n-2, h} \tilde{\Pi}_{h}^{n-2} \varrho \\
& =-\Pi_{h}^{n} \mathrm{~d}_{n-2} \Pi_{h}^{n-1} S_{n-2} \varrho=-\Pi_{h}^{n} \mathrm{~d}_{n-1} \Pi_{h}^{n-1} \tau=-\Pi_{h}^{n} \mathrm{~d}_{n-1} \tau \\
& \quad=-\Pi_{h}^{n}\left(\omega+\Pi_{h}^{n} S_{n-1} \tilde{\Pi}_{h}^{n-1} \eta\right)=-\omega-S_{n-1, h} \tilde{\Pi}_{h}^{n-1} \eta .
\end{aligned}
$$

Combining, we have $-\Pi_{h}^{n} S_{n-1} \equiv-S_{n-1, h} \sigma=\omega$.

(7) Finally, we prove the norm bound. From the boundedness of $S_{n-1}$ in $L^{2}$, 17 , and step (1),

$$
\left\|\Pi_{h}^{n} S_{n-1} \tilde{\Pi}_{h}^{n-1} \eta\right\| \leq c\left\|S_{n-1} \tilde{\Pi}_{h}^{n-1} \eta\right\| \leq c\left\|\tilde{\Pi}_{h}^{n-1} \eta\right\| \leq c\|\eta\|_{1} \leq c\|\mu\| .
$$

Combining with the bounds in step (3) and (2), this gives $\|\varrho\|_{1} \leq c(\|\omega\|+$ $\|\mu\|)$. From 18, we then have $\left\|\mathrm{d}_{n-2} \tilde{\Pi}_{h}^{n-2} \varrho\right\| \leq c(\|\omega\|+\|\mu\|)$. From 17 and the bound in step (1), $\left\|\tilde{\Pi}_{h}^{n-1} \eta\right\| \leq c\|\eta\|_{1} \leq c\|\mu\|$. In view of the definition of $\sigma$, these two last bounds imply that $\|\sigma\| \leq c(\|\omega\|+\|\mu\|)$, while $\left\|\mathrm{d}_{n-1} \sigma\right\| \leq C\left\|\tilde{\Pi}_{h}^{n} \mathrm{~d}_{n-1} \sigma\right\|=\|\mu\|$, and thus we have the desired bound 22 .

We have thus verified the stability conditions (S1) and (S2), and so obtain the following quasi-optimal error estimate (see [14], [15]).

Theorem 9.2 Suppose $(\sigma, u, p)$ is the solution of the elasticity system 11 and $\left(\sigma_{h}, u_{h}, p_{h}\right)$ is the solution of discrete system 12, where the finite element spaces satisfy the hypotheses of Theorem 9.1. Then there is a constant $C$, independent of $h$, such that

$\left\|\sigma-\sigma_{h}\right\|_{H \Lambda}+\left\|u-u_{h}\right\|+\left\|p-p_{h}\right\| \leq C \inf \left(\|\sigma-\tau\|_{H \Lambda}+\|u-v\|+\|p-q\|\right)$,

where the infimum is over all $\tau \in \Lambda_{h}^{n-1}(\mathbb{V}), v \in \Lambda_{h}^{n}(\mathbb{V})$, and $q \in \Lambda_{h}^{n}(\mathbb{K})$.

\section{Refined error estimates}

To see more precisely the contribution to the error from each of the approximating subspaces, we now follow the theory developed in [18] and [20] for error estimates for mixed finite element methods. Since the derivation is fairly simple and we are in an intermediate case to the general theory developed in the references above, we present the complete derivation for the problem we are considering.

Theorem 10.1 Suppose $(\sigma, u, p)$ is the solution of the elasticity system 11 and $\left(\sigma_{h}, u_{h}, p_{h}\right)$ is the solution of discrete system 12, where the finite element subspaces satisfy the hypotheses of Theorem 9.1. Then

$$
\begin{gathered}
\left\|\sigma-\sigma_{h}\right\|+\left\|p-p_{h}\right\|+\left\|u_{h}-\tilde{\Pi}_{h}^{n} u\right\| \leq C\left(\left\|\sigma-\tilde{\Pi}_{h}^{n-1} \sigma\right\|+\left\|p-\Pi_{h}^{n} p\right\|\right), \\
\left\|u-u_{h}\right\| \leq C\left(\left\|\sigma-\tilde{\Pi}_{h}^{n-1} \sigma\right\|+\left\|p-\Pi_{h}^{n} p\right\|+\left\|u-\tilde{\Pi}_{h}^{n} u\right\|\right), \\
\left\|\mathrm{d}_{n-1}\left(\sigma-\sigma_{h}\right)\right\|=\left\|\mathrm{d}_{n-1} \sigma-\tilde{\Pi}_{h}^{n} \mathrm{~d}_{n-1} \sigma\right\| .
\end{gathered}
$$


Proof. Subtracting the equations in 12 from the corresponding equations in 11 , and adding and subtracting appropriate interpolants, we get the error equations

$$
\begin{gathered}
\left\langle A\left(\sigma_{h}-\tilde{\Pi}_{h}^{n-1} \sigma\right), \tau\right\rangle+\left\langle\mathrm{d} \tau, u_{h}-\tilde{\Pi}_{h}^{n} u\right\rangle-\left\langle S \tau, p_{h}-\Pi_{h}^{n} p\right\rangle \\
=\left\langle A\left(\sigma-\tilde{\Pi}_{h}^{n-1} \sigma\right), \tau\right\rangle+\left\langle\mathrm{d} \tau, u-\tilde{\Pi}_{h}^{n} u\right\rangle-\left\langle S \tau, p-\Pi_{h}^{n} p\right\rangle, \quad \tau \in \Lambda_{h}^{n-1}(\mathbb{V}), \\
\left\langle\mathrm{d}\left(\sigma_{h}-\tilde{\Pi}_{h}^{n-1} \sigma\right), v\right\rangle=\left\langle\mathrm{d}\left(\sigma-\tilde{\Pi}_{h}^{n-1} \sigma\right), v\right\rangle, \quad v \in \Lambda_{h}^{n}(\mathbb{V}), \\
\left\langle S\left(\sigma_{h}-\tilde{\Pi}_{h}^{n-1} \sigma\right), q\right\rangle=\left\langle S\left(\sigma-\tilde{\Pi}_{h}^{n-1} \sigma\right), q\right\rangle, \quad q \in \Lambda_{h}^{n}(\mathbb{K}),
\end{gathered}
$$

where we use $\mathrm{d}$ as an abbreviation for $\mathrm{d}_{n-1}$. Now by $16,\left\langle\mathrm{~d}\left(\sigma-\tilde{\Pi}_{h}^{n-1} \sigma\right), v\right\rangle=0$ for $v \in \Lambda_{h}^{n}(\mathbb{V})$ and hence by $18, \mathrm{~d}\left(\sigma_{h}-\tilde{\Pi}_{h}^{n-1} \sigma\right)=0$. Setting

$$
\tau=\sigma_{h}-\tilde{\Pi}_{h}^{n-1} \sigma, \quad v=u_{h}-\tilde{\Pi}_{h}^{n} u, \quad q=p_{h}-\Pi_{h}^{n} p,
$$

and adding the equations, we get

$$
\begin{aligned}
& C\left\|\sigma_{h}-\tilde{\Pi}_{h}^{n-1} \sigma\right\|^{2} \leq\left\langle A\left(\sigma_{h}-\tilde{\Pi}_{h}^{n-1} \sigma\right), \sigma_{h}-\tilde{\Pi}_{h}^{n-1} \sigma\right\rangle \\
& \quad=\left\langle A\left(\sigma-\tilde{\Pi}_{h}^{n-1} \sigma\right), \sigma_{h}-\tilde{\Pi}_{h}^{n-1} \sigma\right\rangle-\left\langle S\left(\sigma_{h}-\tilde{\Pi}_{h}^{n-1} \sigma\right), p-\Pi_{h}^{n} p\right\rangle .
\end{aligned}
$$

Applying standard estimates, we then obtain

$$
\left\|\sigma_{h}-\tilde{\Pi}_{h}^{n-1} \sigma\right\| \leq C\left(\left\|\sigma-\tilde{\Pi}_{h}^{n-1} \sigma\right\|+\left\|p-\Pi_{h}^{n} p\right\|\right),
$$

and hence,

$$
\left\|\sigma-\sigma_{h}\right\| \leq C\left(\left\|\sigma-\tilde{\Pi}_{h}^{n-1} \sigma\right\|+\left\|p-\Pi_{h}^{n} p\right\|\right) .
$$

Next applying Theorem 9.1, with $\omega=p_{h}-\Pi_{h}^{n} p$ and $\mu=u_{h}-\tilde{\Pi}_{h}^{n} u$, we can find $\tau \in \Lambda_{h}^{n-1}(\mathbb{V})$ such that

$$
\begin{gathered}
\tilde{\Pi}_{h}^{n} d \tau=u_{h}-\tilde{\Pi}_{h}^{n} u, \quad-S_{n-1, h} \tau \equiv-\Pi_{h}^{n} S_{n-1} \tau=p_{h}-\Pi_{h}^{n} p, \\
\|\tau\|_{H \Lambda} \leq c\left(\left\|p_{h}-\Pi_{h}^{n} p\right\|+\left\|u_{h}-\tilde{\Pi}_{h}^{n} u\right\|\right) .
\end{gathered}
$$

Making this choice of $\tau$ in 23, we get

$$
\begin{gathered}
\left\|p_{h}-\Pi_{h}^{n} p\right\|^{2}+\left\|u_{h}-\tilde{\Pi}_{h}^{n} u\right\|^{2}=\left\langle A\left(\sigma-\tilde{\Pi}_{h}^{n-1} \sigma\right), \tau\right\rangle-\left\langle A\left(\sigma_{h}-\tilde{\Pi}_{h}^{n-1} \sigma\right), \tau\right\rangle \\
\quad+\left\langle u_{h}-\tilde{\Pi}_{h}^{n} u, u-\tilde{\Pi}_{h}^{n} u\right\rangle+\left\langle p_{h}-\Pi_{h}^{n} p, p-\Pi_{h}^{n} p\right\rangle \\
=\left\langle A\left(\sigma-\tilde{\Pi}_{h}^{n-1} \sigma\right), \tau\right\rangle-\left\langle A\left(\sigma_{h}-\tilde{\Pi}_{h}^{n-1} \sigma\right), \tau\right\rangle+\left\langle p_{h}-\Pi_{h}^{n} p, p-\Pi_{h}^{n} p\right\rangle .
\end{gathered}
$$

Applying standard estimates and 24, we easily obtain

$$
\begin{array}{r}
\left\|p_{h}-\Pi_{h}^{n} p\right\|+\left\|u_{h}-\tilde{\Pi}_{h}^{n} u\right\| \leq C\left(\left\|\sigma-\tilde{\Pi}_{h}^{n-1} \sigma\right\|+\left\|\sigma_{h}-\tilde{\Pi}_{h}^{n-1} \sigma\right\|+\left\|p-\Pi_{h}^{n} p\right\|\right) \\
\leq C\left(\left\|\sigma-\tilde{\Pi}_{h}^{n-1} \sigma\right\|+\left\|p-\Pi_{h}^{n} p\right\|\right) .
\end{array}
$$

Hence, 


$$
\begin{gathered}
\left\|p-p_{h}\right\| \leq C\left(\left\|\sigma-\tilde{\Pi}_{h}^{n-1} \sigma\right\|+\left\|p-\Pi_{h}^{n} p\right\|\right), \\
\left\|u-u_{h}\right\| \leq C\left(\left\|\sigma-\tilde{\Pi}_{h}^{n-1} \sigma\right\|+\left\|p-\Pi_{h}^{n} p\right\|+\left\|u-\tilde{\Pi}_{h}^{n} u\right\|\right) .
\end{gathered}
$$

Finally, since $\left\langle\mathrm{d}\left(\sigma-\sigma_{h}, v\right\rangle=0\right.$ for $v \in \Lambda_{h}^{n}(\mathbb{V})$, we get $\mathrm{d} \sigma_{h}=\tilde{\Pi}_{h}^{n} \mathrm{~d} \sigma$, which establishes the last estimate of the theorem.

\section{Examples of stable finite element methods for the weak symmetry formulation of elasticity}

The examples that follow are of two types. In the first two subsections, we present choices of finite element spaces for which diagram (15) is satisfied without the need for the additional projection $\Pi_{h}^{n}$ in the top sequence. These methods make use of multiple copies of finite element spaces normally associated to the use of mixed methods for scalar second order elliptic problems. In the final three subsections, we consider methods which require the additional projection $\Pi_{h}^{n}$ in the top sequence in diagram (15). This is because the two spaces in the top sequence are ones normally associated to stable pairs for the approximation of the stationary Stokes equations.

\subsection{Arnold, Falk, Winther families}

In the approach of $[8,6,9]$, the spaces are chosen for $r \geq 0$ to be:

$$
\begin{gathered}
\Lambda_{h}^{n-2}(\mathbb{V})=\mathcal{P}_{r+2}^{-} \Lambda^{n-2}\left(\mathcal{T}_{h}\right), \quad \Lambda_{h}^{n-1}(\mathbb{V})=\mathcal{P}_{r+1} \Lambda^{n-1}\left(\mathcal{T}_{h} ; \mathbb{V}\right), \\
\Lambda_{h}^{n}(\mathbb{V})=\mathcal{P}_{r} \Lambda^{n}\left(\mathcal{T}_{h} ; \mathbb{V}\right), \\
\Lambda_{h}^{n-1}(\mathbb{K})=\mathcal{P}_{r+1}^{-} \Lambda^{n-1}\left(\mathcal{T}_{h} ; \mathbb{K}\right), \quad \Lambda_{h}^{n}(\mathbb{K})=\mathcal{P}_{r} \Lambda^{n}\left(\mathcal{T}_{h} ; \mathbb{K}\right)
\end{gathered}
$$

The sequences

$$
\begin{gathered}
\mathcal{P}_{r+1}^{-} \Lambda^{n-1}\left(\mathcal{T}_{h} ; \mathbb{K}\right) \stackrel{\mathrm{d}_{n-1}}{\longrightarrow} \mathcal{P}_{r} \Lambda^{n}\left(\mathcal{T}_{h} ; \mathbb{K}\right) \rightarrow 0 \\
\mathcal{P}_{r+2}^{-} \Lambda^{n-2}\left(\mathcal{T}_{h} ; \mathbb{V}\right) \stackrel{\mathrm{d}_{n-2}}{\longrightarrow} \mathcal{P}_{r+1} \Lambda^{n-1}\left(\mathcal{T}_{h} ; \mathbb{V}\right) \stackrel{\mathrm{d}_{n-1}}{\longrightarrow} \mathcal{P}_{r} \Lambda^{n}\left(\mathcal{T}_{h} ; \mathbb{V}\right) \rightarrow 0
\end{gathered}
$$

are the final parts of longer exact sequences involving the $\mathcal{P}_{r}$ and $\mathcal{P}_{r}^{-}$spaces. Hence, 15 is satisfied without the additional projection at the end of the first sequence. For these spaces, the canonical projection operators $\Pi_{h}^{n-1}$, $\Pi_{h}^{n}, \tilde{\Pi}_{h}^{n-1}$, and $\tilde{\Pi}_{h}^{n}$ satisfy conditions 16 and 17 . Although the canonical projection operator $\tilde{\Pi}_{h}^{n-2}$ does not satisfy 18 , since this operator is not defined on functions in $H^{1} \Lambda^{n-2}(\mathbb{V})$, we can define a modification of this operator, $\tilde{P}_{h}: \Lambda^{n-2}(\Omega ; \mathbb{V}) \rightarrow \mathcal{P}_{r+2}^{-} \Lambda^{n-2}\left(\mathcal{T}_{h} ; \mathbb{V}\right)$ that does satisfy 18 . The operator $\tilde{P}_{h} \omega$ will have the same moments as $\omega$ on faces of codimension 0 and 1 , but with moments of a smoothed approximation of $\omega$ on the faces of codimension 2 . When $n=2$, the issue is simply that the vertex values are not defined and 
this can be remedied by using the ideas of the interpolant of Clement. When $n=3$, additional details are provided in [6]. Thus, to satisfy the hypotheses of Theorem 9.1, it remains to show that

$$
\Pi_{h}^{n-1} S_{n-2} \tilde{P}_{h}=\Pi_{h}^{n-1} S_{n-2} .
$$

This is equivalent to showing that

$$
\Pi_{h}^{n-1} S_{n-2} \omega=0, \quad \forall \omega=\left(I-\tilde{P}_{h}\right) \sigma, \quad \sigma \in \Lambda^{n-2}(\mathbb{V}) .
$$

Since $\tilde{P}_{h} \omega=0$, we have for $n-1 \leq d \leq \min (n, r+n-1)$,

$$
\int_{f} \operatorname{Tr}_{f} \omega \wedge \zeta=0, \quad \zeta \in \mathcal{P}_{r-d+n-1} \Lambda^{d-n+2}(f ; \mathbb{V}), \quad f \in \Delta_{d}\left(\mathcal{T}_{h}\right) .
$$

Note that we have not included similar statements for the vertex degrees of freedom when $n=2$ or the edge degrees of freedom when $n=3$, since we will not need them here. We must show that 25 implies that for $n-1 \leq d \leq$ $\min (n, r+n-1)$,

$$
\int_{f} \operatorname{Tr}_{f} S_{n-2} \omega \wedge \mu=0, \quad \mu \in \mathcal{P}_{r-d+n-1} \Lambda^{d-n+1}(f ; \mathbb{K}), \quad f \in \Delta_{d}\left(\mathcal{T}_{h}\right) .
$$

The simplest case is when $r=0$. When $n=2,25$ becomes

$$
\int_{f} \operatorname{Tr}_{f} \omega \wedge \zeta=0, \quad \zeta \in \mathcal{P}_{0} \Lambda^{1}(f ; \mathbb{V}), \quad f \in \Delta_{1}\left(\mathcal{T}_{h}\right),
$$

which for $\omega=\left(w_{1}, w_{2}\right)^{T}$, is simply the condition

$$
\int_{e} w_{i} \mathrm{~d} e=0, \quad i=1,2, \quad e \in \Delta_{1}\left(\mathcal{T}_{h}\right) .
$$

We then require that

$$
\int_{e} \operatorname{Tr}_{e}\left(-w_{2} \chi \mathrm{d} x_{1}+w_{1} \chi \mathrm{d} x_{2}\right)=0, \quad e \in \Delta_{1}\left(\mathcal{T}_{h}\right) .
$$

But if $\left(t^{1}, t^{2}\right)$ is the unit tangent to $e$, then by 26 ,

$$
\int_{e} \operatorname{Tr}_{e}\left(-w_{2} \chi \mathrm{d} x_{1}+w_{1} \chi \mathrm{d} x_{2}\right)=\int_{e}\left(-w_{2} t^{1}+w_{1} t^{2}\right) \chi \mathrm{d} e=0 .
$$

An analogous argument works for general $r$ when $n=2$, and the basic outline of the proof is the same when $n=3$, although in this case the operator $S_{1}$ is more complicated. The details can be found in [6].

Using Theorem 10.1, it is straightforward to derive the following error estimates, valid for $1 \leq k \leq r+1$, assuming that $\sigma, p$, and $u$ are sufficiently smooth.

$$
\begin{gathered}
\left\|\sigma-\sigma_{h}\right\|+\left\|p-p_{h}\right\|+\left\|u_{h}-\tilde{\Pi}_{h}^{n} u\right\| \leq C h^{k}\left(\|\sigma\|_{k}+\|p\|_{k}\right), \\
\left\|u-u_{h}\right\| \leq C h^{k}\left(\|\sigma\|_{k}+\|p\|_{k}+\|u\|_{k}\right), \quad\left\|\mathrm{d}_{n-1}\left(\sigma-\sigma_{h}\right)\right\| \leq C h^{k}\left\|\mathrm{~d}_{n-1} \sigma\right\|_{k} .
\end{gathered}
$$




\subsection{Arnold, Falk, Winther reduced elements}

In the reduced elements proposed in [8] (in two dimensions) and [6] (in three dimensions), the spaces $\Lambda_{h}^{n}(\mathbb{V}), \Lambda_{h}^{n-1}(\mathbb{K})$, and $\Lambda_{h}^{n}(\mathbb{K})$ remain as chosen above, while the spaces $\Lambda_{h}^{n-2}(\mathbb{V})$ and $\Lambda_{h}^{n-1}(\mathbb{V})$ are modified. Thus, the reduced elements have a somewhat simpler stress space than the methods described above. The basic idea is that in the verification of condition 21 in the last section, we did not use all the degrees of freedom of the space $\mathcal{P}_{2}^{-} \Lambda^{0}\left(\mathcal{T}_{h}\right)$, i.e., we did not use the vanishing of the edge integral of both components of $\omega$, but only the combination $-w_{2} t^{1}+w_{1} t^{2}$ (the normal component). Hence, instead of the vector-valued quadratic space $\mathcal{P}_{2} \Lambda^{0}\left(\mathcal{T}_{h}, \mathbb{V}\right)$, we can use the reduced space obtained from it by imposing the constraint that the tangential component on each edge vary only linearly on that edge. This space of vector fields, which we denote by $\mathcal{P}_{2-} \Lambda^{0}\left(\mathcal{T}_{h}, \mathbb{V}\right)$ has been used previously to approximate the velocity field in the approximation of the stationary Stokes equations (cf. [23, p. 134 ff., 153 ff.]). Together with piecewise constants, it gives a stable finite element approximation scheme for the Stokes equations. An element in this space is determined by its vertex values and the integral of its normal component on each edge. In order to complete the construction, we must provide a vector-valued discrete de Rham sequence in which the space of 0 -forms is $\mathcal{P}_{2-} \Lambda^{0}\left(\mathcal{T}_{h} ; \mathbb{R}^{2}\right)$. This will be the sequence

$$
\mathcal{P}_{2-} \Lambda^{0}\left(\mathcal{T}_{h} ; \mathbb{V}\right) \stackrel{\mathrm{d}_{0}}{\longrightarrow} \mathcal{P}_{1-} \Lambda^{1}\left(\mathcal{T}_{h} ; \mathbb{V}\right) \stackrel{\mathrm{d}_{1}}{\longrightarrow} \mathcal{P}_{0} \Lambda^{2}\left(\mathcal{T}_{h} ; \mathbb{V}\right) \rightarrow 0,
$$

where it remains to define $\mathcal{P}_{1-} \Lambda^{1}\left(\mathcal{T}_{h} ; \mathbb{V}\right)$. This will be the set of $\tau \in$ $\mathcal{P}_{1} \Lambda^{1}\left(\mathcal{T}_{h} ; \mathbb{V}\right)$ for which $\operatorname{Tr}_{e}(\tau) \cdot t$ is constant on any edge $e$ with unit tangent $t$ and unit normal $n$. More specifically, for $\tau \in \mathcal{P}_{1} \Lambda^{1}\left(\mathcal{T}_{h} ; \mathbb{R}^{2}\right), \operatorname{Tr}_{e}(\tau)$ is a vectorvalued 1-form on $e$ of the form $g \mathrm{~d} s$ with $\mu: e \rightarrow \mathbb{R}^{2}$ linear and $\mathrm{d} s$ the volume form-i.e., length form - on $e$. If $\mu \cdot t$ is constant, then $\tau \in \mathcal{P}_{1}^{-} \Lambda^{1}\left(\mathcal{T}_{h} ; \mathbb{V}\right)$. The natural degrees of freedom for this space are the integral and first moment of $\operatorname{Tr}_{e}(\tau) \cdot n$ and the integral of $\operatorname{Tr}_{e}(\tau) \cdot t$. If we use 10 to identify vector-valued 1 -forms and matrix fields, then the condition for a piecewise linear matrix field $W$ to correspond to an element of $\mathcal{P}_{1}^{-} \Lambda^{1}\left(\mathcal{T}_{h} ; \mathbb{R}^{2}\right)$ is that on each edge $e$ with tangent $t$ and normal $n, W n \cdot t$ must be constant on $e$. This defines the reduced space $\Sigma_{h}$, with three degrees of freedom per edge. Together with piecewise constant for displacements and multipliers, this furnishes a stable choice of elements.

A three-dimensional simplified element can be constructed using a similar approach. We start from the space $\mathcal{P}_{2}^{-} \Lambda\left(\mathcal{T}_{h} ; \mathbb{V}\right)$ and see that we do not use all the degrees of freedom to satisfy condition 21 . We thus define a reduced space $\mathcal{P}_{2-}^{-} \Lambda\left(\mathcal{T}_{h} ; \mathbb{V}\right)$ and a space $\mathcal{P}_{1-} \Lambda^{2}\left(\mathcal{T}_{h} ; \mathbb{V}\right)$ such that these spaces, together with $\mathcal{P}_{0} \Lambda^{3}\left(\mathcal{T}_{h} ; \mathbb{V}\right)$, form the exact sequence

$$
\mathcal{P}_{2-} \Lambda^{1}\left(\mathcal{T}_{h} ; \mathbb{V}\right) \stackrel{\mathrm{d}_{1}}{\longrightarrow} \mathcal{P}_{1-} \Lambda^{2}\left(\mathcal{T}_{h} ; \mathbb{V}\right) \stackrel{\mathrm{d}_{2}}{\longrightarrow} \mathcal{P}_{0} \Lambda^{3}\left(\mathcal{T}_{h} ; \mathbb{V}\right) \rightarrow 0 .
$$

We are then able to replace the space $\mathcal{P}_{1} \Lambda^{1}\left(\mathcal{T}_{h} ; \mathbb{V}\right)$, which has 36 degrees of freedom (9 per face), by the space $\mathcal{P}_{1-} \Lambda^{2}\left(\mathcal{T}_{h} ; \mathbb{V}\right)$, which has 24 degrees of 
freedom ( 6 per face). If we identify an element in our reduced space with a matrix $W$ is the manner discussed previously, then we get on each face the six degrees of freedom:

$\int_{f} W n \mathrm{~d} f, \int_{f}(x \cdot t) n^{T} W n \mathrm{~d} f, \int_{f}(x \cdot s) n^{T} W n \mathrm{~d} f, \int_{f}\left[(x \cdot t) s^{T}-(x \cdot s) t^{T}\right] W n \mathrm{~d} f$,

where $s$ and $t$ denote orthogonal unit tangent vectors on the face $f$. More details can be found in [6].

\subsection{PEERS}

In the PEERS method [3], $n=2$ and we choose

$$
\begin{gathered}
\Lambda_{h}^{1}(\mathbb{V})=\mathcal{P}_{1}^{-} \Lambda^{1}\left(\mathcal{T}_{h} ; \mathbb{V}\right)+d B_{3} \Lambda^{0}\left(\mathcal{T}_{h} ; \mathbb{V}\right), \quad \Lambda_{h}^{2}(\mathbb{V})=\mathcal{P}_{0} \Lambda^{2}\left(\mathcal{T}_{h} ; \mathbb{V}\right), \\
\Lambda_{h}^{2}(\mathbb{K})=\mathcal{P}_{1} \Lambda^{2}\left(\mathcal{T}_{h} ; \mathbb{K}\right) \cap H^{1} \Lambda^{2}(\mathbb{K}), \quad \text { which we denote by } \mathcal{P}_{1}^{0} \Lambda^{2}\left(\mathcal{T}_{h} ; \mathbb{K}\right),
\end{gathered}
$$

where $B_{3}$ denotes the space of cubic bubble functions. We then choose the two remaining spaces as

$$
\Lambda_{h}^{0}(\mathbb{V})=\left(\mathcal{P}_{1}+B_{3}\right) \Lambda^{0}\left(\mathcal{T}_{h} ; \mathbb{V}\right), \quad \Lambda_{h}^{1}(\mathbb{K})=S_{0} \Lambda_{h}^{0}(\mathbb{V}) .
$$

It is easy to see that

$$
\Lambda_{h}^{1}(\mathbb{K})=\left(\mathcal{P}_{1}+B_{3}\right) \Lambda^{1}\left(\mathcal{T}_{h} ; \mathbb{K}\right) \cap H^{1} \Lambda^{1}(\mathbb{K}) \equiv\left(\mathcal{P}_{1}^{0}+B_{3}\right) \Lambda^{1}\left(\mathcal{T}_{h} ; \mathbb{K}\right) .
$$

Since the sequence

$$
\mathcal{P}_{1} \Lambda^{0}\left(\mathcal{T}_{h} ; \mathbb{V}\right) \stackrel{\mathrm{d}_{0}}{\longrightarrow} \mathcal{P}_{1}^{-} \Lambda^{1}\left(\mathcal{T}_{h} ; \mathbb{V}\right) \stackrel{\mathrm{d}_{1}}{\longrightarrow} \mathcal{P}_{0} \Lambda^{2}\left(\mathcal{T}_{h} ; \mathbb{V}\right) \rightarrow 0
$$

is exact, so is the sequence

$$
\left(\mathcal{P}_{1}+B_{3}\right) \Lambda^{0}\left(\mathcal{T}_{h} ; \mathbb{V}\right) \stackrel{\mathrm{d}_{0}}{\longrightarrow} \mathcal{P}_{1}^{-} \Lambda^{1}\left(\mathcal{T}_{h} ; \mathbb{V}\right)+\mathrm{d}_{0} B_{3} \Lambda^{0}\left(\mathcal{T}_{h} ; \mathbb{V}\right) \stackrel{\mathrm{d}_{1}}{\longrightarrow} \mathcal{P}_{0} \Lambda^{2}\left(\mathcal{T}_{h} ; \mathbb{V}\right) \rightarrow 0 .
$$

For this choice of spaces, however, it is not true that $d \Lambda_{h}^{1}(\mathbb{K})=\Lambda_{h}^{2}(\mathbb{K})$. Instead, we use the more general condition $\Pi_{h}^{2} d \Lambda_{h}^{1}(\mathbb{K})=\Lambda_{h}^{2}(\mathbb{K})$, which allows the use of stable Stokes elements. The proof that the combination $\left(\mathcal{P}_{1}^{0}+B_{3}\right) \Lambda^{1}\left(\mathcal{T}_{h} ; \mathbb{K}\right)$ and $\mathcal{P}_{1}^{0} \Lambda^{2}\left(\mathcal{T}_{h} ; \mathbb{K}\right)$ is a stable Stokes pair (the Minielement) involves construction of an interpolation operator $\Pi_{h}^{1}: H^{1} \Lambda^{1}(\mathbb{K}) \mapsto$ $\left(\mathcal{P}_{1}^{0}+B_{3}\right) \Lambda^{1}\left(\mathcal{T}_{h} ; \mathbb{K}\right)$ satisfying

$$
\left\langle\mathrm{d}_{1}\left(\tau-\Pi_{h}^{1} \tau\right), q_{h}\right\rangle=0, q_{h} \in \Lambda_{h}^{2}(\mathbb{K}), \quad\left\|\Pi_{h}^{1} \tau\right\|_{1} \leq C\|\tau\|_{1}, \tau \in H^{1} \Lambda^{1}(\mathbb{K}),
$$

which gives properties 16 and 17 for the operators $\Pi_{h}^{1}$ and $\Pi_{h}^{2}$. Properties 16 and 17 for the operators $\tilde{\Pi}_{h}^{1}$ and $\tilde{\Pi}_{h}^{2}$ are satisfied by the Raviart-Thomas interpolant $\tilde{\Pi}_{h}^{1}: H^{1} \Lambda^{1}(\mathbb{V}) \mapsto \mathcal{P}_{1}^{-} \Lambda^{1}\left(\mathcal{T}_{h} ; \mathbb{V}\right)$. Finally, one can easily check that 18 and 21 are satisfied if we define 


$$
\tilde{\Pi}_{h}^{0}: H^{1} \Lambda^{0}(\mathbb{V}) \mapsto\left(\mathcal{P}_{1}+B_{3}\right) \Lambda^{0}\left(\mathcal{T}_{h} ; \mathbb{V}\right)
$$

by

$$
\tilde{\Pi}_{h}^{0} \tau=S_{0}^{-1} \Pi_{h}^{1} S_{0} \tau .
$$

Note that condition 21 is then trivial, since for $\tau \in H^{1} \Lambda^{0}(\mathbb{V})$,

$$
S_{0, h} \tilde{\Pi}_{h}^{0} \tau=\Pi_{h}^{1} S_{0} S_{0}^{-1} \Pi_{h}^{1} S_{0} \tau=\Pi_{h}^{1} S_{0} \tau .
$$

Applying Theorem 10.1, and standard approximation and regularity results, we obtain the error estimates

$$
\left\|\sigma-\sigma_{h}\right\|_{0}+\left\|p-p_{h}\right\|_{0}+\left\|u-u_{h}\right\|_{0} \leq C h\left(\|\sigma\|_{1}+\|p\|_{1}+\|u\|_{1}\right) \leq C h\|f\|_{0} .
$$

\subsection{A PEERS-like method with improved stress approximation}

In this new method, we change one of the spaces used in the PEERS element and both of the auxiliary spaces used in the analysis, i.e., we choose

$$
\Lambda_{h}^{1}(\mathbb{V})=\mathcal{P}_{1} \Lambda^{1}\left(\mathcal{T}_{h} ; \mathbb{V}\right), \quad \Lambda_{h}^{2}(\mathbb{V})=\mathcal{P}_{0} \Lambda^{2}\left(\mathcal{T}_{h} ; \mathbb{V}\right), \quad \Lambda_{h}^{2}(\mathbb{K})=\mathcal{P}_{1}^{0} \Lambda^{2}\left(\mathcal{T}_{h} ; \mathbb{K}\right),
$$

and the two remaining spaces as

$$
\Lambda_{h}^{0}(\mathbb{V})=\mathcal{P}_{2} \Lambda^{0}\left(\mathcal{T}_{h} ; \mathbb{V}\right), \quad \Lambda_{h}^{1}(\mathbb{K})=S_{0} \Lambda_{h}^{0}(\mathbb{V}) \equiv \mathcal{P}_{2} \Lambda^{1}\left(\mathcal{T}_{h} ; \mathbb{K}\right) \cap H^{1} \Lambda^{1}(\mathbb{K}) .
$$

The basic change from the analysis of the PEERS element is that we use the fact that the combination of $\mathcal{P}_{2} \Lambda^{1}\left(\mathcal{T}_{h} ; \mathbb{K}\right) \cap H^{1} \Lambda^{1}(\mathbb{K})$ and $\mathcal{P}_{1}^{0} \Lambda^{2}\left(\mathcal{T}_{h} ; \mathbb{K}\right)$ is a stable pair of spaces for the Stokes problem (i.e., the Taylor-Hood element).

We may also view this new method as a modification of the lowest order Arnold-Falk-Winther method, where we are using the same stress and displacement spaces and lower exact sequence as in that method, but have changed the spaces with values in $\mathbb{K}$. The advantage of this modification is that it produces a higher order approximation to the stress variable. Looking at the error estimates given in Theorem 10.1, we see that the error estimate for $\left\|\sigma-\sigma_{h}\right\|_{0}$ depends both on $\left\|\sigma-\tilde{\Pi}_{h}^{n-1} \sigma\right\|_{0}$ and $\left\|p-\Pi_{h}^{n} p\right\|_{0}$. In the lowest order Arnold-Falk-Winther method, $\left\|\sigma-\tilde{\Pi}_{h}^{n-1} \sigma\right\|_{0} \leq C h^{2}\|\sigma\|_{2}$, since we are using $\mathcal{P}_{1}$ elements to approximate $\sigma$. The fact that piecewise constants are used to approximate the multiplier results in only an $O(h)$ approximation for the second term. By using linear elements in the modified method, we recover second order convergence. Since we use only piecewise constants to approximate $u$, we can only obtain the estimate $\left\|u-u_{h}\right\|_{0} \leq C h$. However, since the quantity $\left\|u_{h}-\tilde{\Pi}_{h}^{n} u\right\|_{0}$ is also $O\left(h^{2}\right)$, we might be able to obtain a better result by a post-processing procedure.

Remark 1. We note that some of these same ideas have been used to develop hybrid methods for the approximation of the elasticity equations. For example, see $[21]$. 


\subsection{Methods of Stenberg}

A family of methods proposed and analyzed by Stenberg [28] chooses for $r \geq 2$, $n=2$ or $n=3$,

$$
\begin{gathered}
\Lambda_{h}^{n-1}(\mathbb{V})=\mathcal{P}_{r} \Lambda^{n-1}\left(\mathcal{T}_{h} ; \mathbb{V}\right)+d B_{r+n} \Lambda^{n-2}\left(\mathcal{T}_{h} ; \mathbb{V}\right), \quad \Lambda_{h}^{n}(\mathbb{V})=\mathcal{P}_{r-1} \Lambda^{n}\left(\mathcal{T}_{h} ; \mathbb{V}\right), \\
\Lambda_{h}^{n}(\mathbb{K})=\mathcal{P}_{r} \Lambda^{n}\left(\mathcal{T}_{h} ; \mathbb{K}\right),
\end{gathered}
$$

where $B_{r+n}$ denotes the space of functions which on each simplex $T$ have the form $b_{T} \mathcal{P}_{r-1}$, where $b_{T}(x)=\prod_{i=1}^{n+1} \lambda_{i}(x)$, i.e., the space of bubbles of degree $r+n$. To fit our framework, we then choose the two remaining spaces as

$$
\begin{gathered}
\Lambda_{h}^{n-2}(\mathbb{V})=\left(\mathcal{P}_{r+1}+B_{r+n}\right) \Lambda^{n-2}\left(\mathcal{T}_{h} ; \mathbb{V}\right), \\
\Lambda_{h}^{n-1}(\mathbb{K})=\left(\mathcal{P}_{r+1}+B_{r+n}\right) \Lambda^{n-1}\left(\mathcal{T}_{h} ; \mathbb{K}\right) \cap H^{1} \Lambda^{1}(\mathbb{K}) .
\end{gathered}
$$

Since the sequence

$$
\mathcal{P}_{r+1} \Lambda^{n-2}\left(\mathcal{T}_{h} ; \mathbb{V}\right) \stackrel{\mathrm{d}_{n-2}}{\longrightarrow} \mathcal{P}_{r} \Lambda^{n-1}\left(\mathcal{T}_{h} ; \mathbb{V}\right) \stackrel{\mathrm{d}_{n-1}}{\longrightarrow} \mathcal{P}_{r-1} \Lambda^{n}\left(\mathcal{T}_{h} ; \mathbb{V}\right) \rightarrow 0
$$

is exact, it is easy to see that the sequence

$$
\begin{aligned}
&\left(\mathcal{P}_{r+1}+B_{r+n}\right) \Lambda^{n-2}\left(\mathcal{T}_{h} ; \mathbb{V}\right) \stackrel{\mathrm{d}_{n-2}}{\longrightarrow} \mathcal{P}_{r} \Lambda^{n-1}\left(\mathcal{T}_{h} ; \mathbb{V}\right)+\mathrm{d}_{n-2} B_{r+n} \Lambda^{n-2}\left(\mathcal{T}_{h} ; \mathbb{V}\right) \\
& \stackrel{\mathrm{d}_{n-1}}{\longrightarrow} \\
& \mathcal{P}_{r-1} \Lambda^{n}\left(\mathcal{T}_{h} ; \mathbb{V}\right) \rightarrow 0
\end{aligned}
$$

will be exact. Again it is not true that $d \Lambda_{h}^{n-1}(\mathbb{K})=\Lambda_{h}^{n}(\mathbb{K})$, and so we use the more general condition,

$$
\Pi_{h}^{n} d \Lambda_{h}^{n-1}(\mathbb{K})=\Lambda_{h}^{n}(\mathbb{K}),
$$

which allows the use of stable Stokes spaces. From the definition of $S_{n-2}$, it it easy to see that when $n=2$,

$$
S_{0} \Lambda_{h}^{0}(\mathbb{V})=\left(\mathcal{P}_{r+1}+B_{r+n}\right) \Lambda^{1}\left(\mathcal{T}_{h} ; \mathbb{K}\right) \cap H^{1} \Lambda^{1}(\mathbb{K}),
$$

and when $n=3$,

$$
S_{1}\left[\Lambda_{h}^{1}(\mathbb{V}) \cap H^{1} \Lambda^{2}(\mathbb{V})\right]=\left(\mathcal{P}_{r+1}+B_{r+n}\right) \Lambda^{2}\left(\mathcal{T}_{h} ; \mathbb{K}\right) \cap H^{1} \Lambda^{2}(\mathbb{K}) .
$$

The proof that the combination $\left(\mathcal{P}_{r+1}+B_{r+n}\right) \Lambda^{n-1}\left(\mathcal{T}_{h} ; \mathbb{K}\right) \cap H^{1} \Lambda^{n-1}(\mathbb{K})$ and $\mathcal{P}_{r} \Lambda^{n}\left(\mathcal{T}_{h} ; \mathbb{K}\right)$ is a stable pair of Stokes elements (cf $[23,15]$ ) gives us precisely what we need to establish 16 and 17 for the operators $\Pi_{h}^{n-1}$ and $\Pi_{h}^{n}$, i.e., the construction of an interpolation operator $\Pi_{h}^{n-1}: H^{1} \Lambda^{n-1}(\mathbb{K}) \mapsto$ $\left(\mathcal{P}_{r+1}+B_{r+n}\right) \Lambda^{n-1}\left(\mathcal{T}_{h} ; \mathbb{K}\right) \cap H^{1} \Lambda^{n-1}(\mathbb{K})$ satisfying

$$
\begin{gathered}
\left\langle\mathrm{d}_{n-1}\left(\tau-\Pi_{h}^{n-1} \tau\right), q_{h}\right\rangle=0, q_{h} \in \Lambda_{h}^{n}(\mathbb{K}), \\
\left\|\Pi_{h}^{n-1} \tau\right\|_{1} \leq C\|\tau\|_{1}, \tau \in H^{1} \Lambda^{n-1}(\mathbb{K}) .
\end{gathered}
$$


Properties 16 and 17 for the operators $\tilde{\Pi}_{h}^{n-1}$ and $\tilde{\Pi}_{h}^{n}$ are satisfied by the canonical canonical interpolant $\tilde{\Pi}_{h}^{n-1}: H^{1} \Lambda^{n-1}(\mathbb{V}) \mapsto \mathcal{P}_{r} \Lambda^{n-1}\left(\mathcal{T}_{h} ; \mathbb{V}\right)$. Finally, it is easy to check that 18 and 21 are satisfied if we define

$$
\tilde{\Pi}_{h}^{n-2}: H^{1} \Lambda^{n-2}(\mathbb{V}) \mapsto\left(\mathcal{P}_{r+1}+B_{r+n}\right) \Lambda^{n-2}\left(\mathcal{T}_{h} ; \mathbb{V}\right) \cap H^{1} \Lambda^{n-2}(\mathbb{V})
$$

by

$$
\tilde{\Pi}_{h}^{n-2} \tau=S_{n-2}^{-1} \Pi_{h}^{n-1} S_{n-2} \tau .
$$

When $n=2$, this same analysis also carries over to the case $r=1$, since the combination $\left(\mathcal{P}_{2}+B_{3}\right) \Lambda^{1}\left(\mathcal{T}_{h} ; \mathbb{K}\right) \cap H^{1} \Lambda^{1}(\mathbb{K})$ and $\mathcal{P}_{1} \Lambda^{2}\left(\mathcal{T}_{h} ; \mathbb{K}\right)$ is a stable pair of Stokes elements. The situation is more complicated in three dimensions, since the analogous combination is not a stable pair of Stokes elements.

Using Theorem 10.1, it is straightforward to derive the following error estimates, assuming that $\sigma, p$, and $u$ are sufficiently smooth.

$$
\begin{gathered}
\left\|\sigma-\sigma_{h}\right\|+\left\|p-p_{h}\right\|+\left\|u_{h}-\tilde{\Pi}_{h}^{n} u\right\| \leq C h^{k}\left(\|\sigma\|_{k}+\|p\|_{k}\right), \quad 1 \leq k \leq r+1, \\
\left\|u-u_{h}\right\| \leq C h^{k}\left(\|\sigma\|_{k}+\|p\|_{k}+\|u\|_{k}\right), \quad 1 \leq k \leq r, \\
\left\|\mathrm{~d}_{n-1}\left(\sigma-\sigma_{h}\right)\right\| \leq C h^{k}\left\|\mathrm{~d}_{n-1} \sigma\right\|_{k}, \quad 1 \leq k \leq r .
\end{gathered}
$$

\section{References}

1. S. Adams and B. Cockburn, A mixed finite element method for elasticity in three dimensions, J. Sci. Comput. 25 (2005), 515-521.

2. M. Amara and J. M. Thomas, Equilibrium finite elements for the linear elastic problem, Numer. Math. 33 (1979), 367-383. MR MR553347 (81b:65096)

3. D. N. Arnold, F. Brezzi, and J. Douglas, Jr., PEERS: a new mixed finite element for plane elasticity, Japan J. Appl. Math. 1 (1984), 347-367. MR MR840802 (87h:65189)

4. D. N. Arnold, J. Douglas, Jr., and C. P. Gupta, A family of higher order mixed finite element methods for plane elasticity, Numer. Math. 45 (1984), 1-22. MR MR761879 (86a:65112)

5. D. N. Arnold and R. S. Falk, A new mixed formulation for elasticity, Numer. Math. 53 (1988), 13-30. MR MR946367 (89f:73020)

6. D. N. Arnold, R. S. Falk, and R. Winther, Mixed finite element methods for linear elasticity with weakly imposed symmetry, Math. Comput. (2005), submitted.

7. _ Differential complexes and stability of finite element methods I: The de Rham complex, Compatible Spatial Discretizations, The IMA Volumes in Mathematics and its Applications, vol. 142, Springer, Berlin, 2006, pp. 23-46.

8. Differential complexes and stability of finite element methods II: The elasticity complex, Compatible Spatial Discretizations, The IMA Volumes in Mathematics and its Applications, vol. 142, Springer, Berlin, 2006, pp. 47-68.

9. __ Finite element exterior calculus, homological techniques, and applications, Acta Numer. (2006), 1-155.

10. D. N. Arnold and R. Winther, Mixed finite elements for elasticity, Numer. Math. 92 (2002), 401-419. MR MR1930384 (2003i:65103) 
11. _ Mixed finite elements for elasticity in the stress-displacement formulation, Current trends in scientific computing (Xi'an, 2002), Contemp. Math., vol. 329, Amer. Math. Soc., Providence, RI, 2003, pp. 33-42. MR MR2022629

12. _ Nonconforming mixed elements for elasticity, Math. Models Methods Appl. Sci. 13 (2003), no. 3, 295-307, Dedicated to Jim Douglas, Jr. on the occasion of his 75 th birthday. MR MR1977627 (2004f:65176)

13. I. N. Bernšteĭn, I. M. Gel'fand, and S. I. Gel'fand, Differential operators on the base affine space and a study of $\mathfrak{g}$-modules, Lie Groups and Their Representations (Proc. Summer School, Bolyai János Math. Soc., Budapest, 1971), Halsted, New York, 1975, pp. 21-64. MR MR0578996 (58 \#28285)

14. F. Brezzi, On the existence, uniqueness and approximation of saddle-point problems arising from Lagrangian multipliers, Rev. Française Automat. Informat. Recherche Opérationnelle Sér. Rouge 8 (1974), 129-151. MR MR0365287 (51 \#1540)

15. F. Brezzi and M. Fortin, Mixed and Hybrid Finite Element Methods, Springer Series in Computational Mathematics, vol. 15, Springer, New York, 1991. MR MR1115205 (92d:65187)

16. A. Čap, J. Slovák, and V. Souček, Bernstein-Gelfand-Gelfand sequences, Ann. of Math. (2) 154 (2001), 97-113. MR MR1847589 (2002h:58034)

17. J. Douglas, Jr., T. Dupont, P. Percell, and L. R. Scott, A family of $C^{1}$ finite elements with optimal approximation properties for various Galerkin methods for 2nd and 4th order problems, RAIRO Anal. Numér. 13 (1979), no. 3, 227-255. MR MR543934 (80k:65087)

18. J. Douglas, Jr. and J. E. Roberts, Global estimates for mixed methods for second order elliptic equations, Math. Comp. 44 (1985), 39-52. MR MR771029 (86b:65122)

19. M. Eastwood, A complex from linear elasticity, Rend. Circ. Mat. Palermo (2) Suppl. (2000), no. 63, 23-29. MR MR1758075 (2001j:58033)

20. R. S. Falk and J. E. Osborn, Error estimates for mixed methods, RAIRO Anal. Numér. 14 (1980), 249-277. MR MR592753 (82j:65076)

21. M. Farhloul and M. Fortin, Dual hybrid methods for the elasticity and the Stokes problems: a unified approach, Numer. Math. 76 (1997), 419-440. MR MR1464150 (98f:65106)

22. B. M. Fraeijs de Veubeke, Stress function approach, Proc. of the World Congress on Finite Element Methods in Structural Mechanics, vol. 1, Bournemouth, Dorset, England, 1975, pp. J.1-J.51.

23. V. Girault and P.-A. Raviart, Finite element methods for Navier-Stokes equations, Springer Series in Computational Mathematics, vol. 5, Springer-Verlag, Berlin, 1986, Theory and algorithms. MR MR851383 (88b:65129)

24. C. Johnson and B. Mercier, Some equilibrium finite element methods for two-dimensional elasticity problems, Numer. Math. 30 (1978), 103-116. MR MR0483904 (58 \#3856)

25. M. E. Morley, A family of mixed finite elements for linear elasticity, Numer. Math. 55 (1989), 633-666. MR MR1005064 (90f:73006)

26. E. Stein and R. Rolfes, Mechanical conditions for stability and optimal convergence of mixed finite elements for linear plane elasticity, Comput. Methods Appl. Mech. Engrg. 84 (1990), 77-95. MR MR1082821 (91i:73045)

27. R. Stenberg, On the construction of optimal mixed finite element methods for the linear elasticity problem, Numer. Math. 48 (1986), 447-462. MR MR834332 (87i:73062) 
28. _ A family of mixed finite elements for the elasticity problem, Numer. Math. 53 (1988), 513-538. MR MR954768 (89h:65192)

29. _ Two low-order mixed methods for the elasticity problem, The Mathematics of Finite Elements and Applications, VI (Uxbridge, 1987), Academic Press, London, 1988, pp. 271-280. MR MR956898 (89j:73074)

30. V. B. Watwood, Jr. and B. J. Hartz, An equilibrium stress field model for finite element solution of two-dimensional elastostatic problems, Internat. J. Solids Structures 4 (1968), 857-873. 